\title{
Gluing and wormholes for the Einstein constraint equations
}

\author{
James Isenberg* \\ University of Oregon
}

\author{
Rafe Mazzeo ${ }^{\dagger}$ \\ Stanford University
}

July 23, 2002

\author{
Daniel Pollack $\ddagger$ \\ University of Washington
}

\begin{abstract}
We establish a general gluing theorem for constant mean curvature solutions of the vacuum Einstein constraint equations. This allows one to take connected sums of solutions or to glue a handle (wormhole) onto any given solution. Away from this handle region, the initial data sets we produce can be made as close as desired to the original initial data sets. These constructions can be made either when the initial manifold is compact or asymptotically Euclidean or asymptotically hyperbolic, with suitable corresponding conditions on the extrinsic curvature. In the compact setting a mild nondegeneracy condition is required. In the final section of the paper, we list a number ways this construction may be used to produce new types of vacuum spacetimes.
\end{abstract}

\section{Introduction}

\subsection{The constraint equations and surgery}

The Einstein equations for the gravitational field on a Lorentzian four-manifold $Z$ form, modulo diffeomorphisms, a locally well-posed hyperbolic system [11], and vacuum solutions may be obtained from a set of Cauchy data on a three-dimensional spacelike slice $\Sigma \subset Z$. A vacuum initial data set for this problem consists of two symmetric tensors $\gamma$ and $\Pi$ on $\Sigma$, which correspond respectively to the induced metric and second fundamental form on $\Sigma$. Thus $\gamma$ is positive definite while $\Pi$ is at least apparently unrestricted. However, in order for the Einstein evolution to exist, at least for a short time, it is necessary and sufficient 11] that these tensors satisfy the constraint equations:

$$
\begin{array}{r}
\operatorname{div} \Pi-\nabla \operatorname{tr} \Pi=0 \\
R_{\gamma}-|\Pi|_{\gamma}^{2}+(\operatorname{tr} \Pi)^{2}=0
\end{array}
$$

Here $\nabla$ is the Levi-Civita connection for $\gamma, R_{\gamma}$ its scalar curvature, and the divergence, div $\gamma$, trace, $\operatorname{tr}_{\gamma}$ and norm squared of $\Pi,|\Pi|_{\gamma}^{2}=\Pi_{a b} \Pi_{c d} \gamma^{a c} \gamma^{b d}$, are all computed with respect to $\gamma$.

\footnotetext{
* Supported by the NSF under Grant PHY-0099373

${ }^{\dagger}$ Supported by the NSF under Grant DMS-9971975 and at MSRI by NSF grant DMS-9701755

${ }^{\ddagger}$ Supported by the NSF under Grant DMS-9704515
} 
Having fixed the three-manifold $\Sigma$, it is not apparent that solutions $(\gamma, \Pi)$ of these constraint equations exist, or how to find them. However, there is a systematic procedure which generates solutions in a large number of cases, which we review now. Let us begin with a pair of tensors $(\gamma, \Pi)$, which do not necessarily satisfy the constraint equations, but which have the property that the mean curvature $\tau=\operatorname{tr}_{\gamma} \Pi$ is constant. The method we are describing relies crucially on this assumption. Decompose $\Pi$ into its trace-free and pure trace parts:

$$
\Pi=\mu+\frac{\tau}{3} \gamma
$$

The first of the constraint equations (11) requires that $\operatorname{div} \mu=0$, since $\tau$ is constant, so that $\mu$ is what is usually called a transverse-traceless (TT) tensor field. The idea is to modify the pair $(\gamma, \Pi)$ by changing $\gamma$ and the trace-free part $\mu$ of $\Pi$ by a conformal factor; by a judicious choice of this factor, the new tensors will satisfy the constraint equations. More specifically, for any $\phi>0$ we set

$$
\begin{aligned}
\tilde{\gamma} & =\phi^{4} \gamma, \\
\tilde{\Pi}=\tilde{\mu}+\frac{\tau}{3} \tilde{\gamma} & =\phi^{-2} \mu+\frac{\tau}{3} \phi^{4} \gamma .
\end{aligned}
$$

The factor $\phi^{-2}$ in $\tilde{\mu}$ is the only one for which $\tilde{\mu}$ is divergence-free with respect to $\tilde{\gamma}$. (Note that $\tilde{\Pi}$ and $\tilde{\mu}$ as they appear in (5) are covariant tensors; the factor $\phi^{-10}$ replaces $\phi^{-2}$ if we work with $\tilde{\Pi}$ and $\tilde{\mu}$ in contravariant form.) This new pair $(\tilde{\gamma}, \tilde{\Pi})$ satisfies the constraint equations provided the conformal factor $\phi$ satisfies the Lichnerowicz equation

$$
\Delta \phi-\frac{1}{8} R \phi+\frac{1}{8}|\mu|^{2} \phi^{-7}-\frac{1}{12} \tau^{2} \phi^{5}=0 .
$$

Here the Laplacian, scalar curvature and norm-squared of $\mu$ are all computed relative to $\gamma$. By well-known analytic techniques one may find solutions of this semilinear equation, and so altogether this procedure produces many admissible pairs of tensors satisfying the constraint equations. In effect, the constancy of the mean curvature $\tau$ decouples the equations (1), (2), reducing them to the facts that $\mu$ is transverse-traceless and $\phi$ satisfies the Lichnerowicz equation. These formulations are equivalent and so we label initial data sets in one of three equivalent ways: as $(\gamma, \Pi)$ if these tensors satisfy the constraint equations, or as $(\gamma, \Pi, \phi)$ if $\phi$ is the conformal factor which alters $(\gamma, \Pi)$ to tensors satisfying the constraint equations, or finally (suppressing $\tau$ ) as $(\gamma, \mu, \phi)$. When $\phi$ is omitted in this notation, then $\phi=1$ is implied.

When $\Sigma$ is compact, complete existence results are available in this CMC case [18]; analogous results are known when $\Sigma$ is either asymptotically flat [7], [15], [9], [8] or asymptotically hyperboloidal [3], [2]. In the non-CMC case, when $\tau$ is no longer constant, only partial results have been obtained [19], [12], [14], [20] but this direction warrants greater attention since the existence of constant mean curvature slices is apparently a restrictive assumption in relativity [5], [6].

In this paper we address the following question. Suppose we are given a three-manifold $\Sigma$ along with an initial data set $(\gamma, \Pi)$ on it which satisfy the constraint equations. Then is it possible to modify $\Sigma$ by 'surgery' to obtain a new, topologically distinct manifold $\Sigma$ ', and to find tensors $\gamma^{\prime}$ and $\Pi^{\prime}$ on $\Sigma^{\prime}$ which themselves satisfy the constraint equations? Since we can find solutions by applying the procedure above to $\Sigma^{\prime}$ in the first place, the 
more precise question we propose to study is whether it is possible to perform the surgery on a geometrically small subset of $\Sigma$ and to find solutions $\left(\gamma^{\prime}, \Pi^{\prime}\right)$ which are very close to the initial solutions $(\gamma, \Pi)$ away from this subset?

Let us phrase this more concretely. There are many different types of surgeries possible on higher dimensional manifolds, and really only two types in three dimensions. One is akin to Dehn surgery, while the other, on which we focus, consists of 'adding a handle' in the following sense. Choose any two points $p_{1}, p_{2} \in \Sigma$ and let $B_{1}$ and $B_{2}$ be small (metric) balls centered at these points. Excise these balls to obtain a manifold $\Sigma^{\prime \prime}=\Sigma \backslash\left(B_{1} \cup B_{2}\right)$. The boundary of $\Sigma^{\prime \prime}$ consists of two copies of $S^{2}$, the same as the product of an interval with a two-sphere, $I \times S^{2}$. Identifying these two manifolds along this common boundary yields a new manifold

$$
\widehat{\Sigma}=\Sigma^{\prime \prime} \bigsqcup_{\{ \pm 1\} \times S^{2}} I \times S^{2}
$$

Informally, we propose to restrict the original initial data set $(\gamma, \Pi)$ to $\Sigma^{\prime \prime}$, modify this data slightly, and then find a one-parameter family of extensions $\left(\gamma_{\varepsilon}, \Pi_{\varepsilon}\right)$ of this data to $\widehat{\Sigma}, \varepsilon \in\left(0, \varepsilon_{0}\right)$, each of which also satisfy the constraint equations. The parameter $\varepsilon$ measures the size of the handle. We shall do this in such a way that on any compact set $K$ away from the handle, $\left(\gamma_{\varepsilon}, \Pi_{\varepsilon}\right)$ converges to $(\gamma, \Pi)$ as some power of $\varepsilon$. The data on the handle will converge to zero. In the compact setting, the only condition needed to make this procedure work is a very mild nondegeneracy condition as well as the assumption that $\Pi \not \equiv 0$; the precise statement is contained in the main theorem below. In particular, we cannot handle compact 'time-symmetric slices', i.e. those with $\Pi \equiv 0$, in which case the constraint equations reduce to the vanishing of scalar curvature, and known topological obstructions (see [32] for example) prevent this sort of construction from working in general then.

There are two main cases of this construction. In the first, $\Sigma$ consists of two components, $\Sigma_{1}$ and $\Sigma_{2}$, and the two points $p_{1}, p_{2}$ lie in these separate components. Then we are producing solutions of the constraint equations on the connected sum $\Sigma_{1} \# \Sigma_{2}$ of these two components. In the other case, $\Sigma$ is connected, and this construction may be regarded as demonstrating that a 'wormhole' can be added to most solutions of the constraint equations. Both cases are of clear physical interest.

The analytic techniques used in the proof here are in the spirit of many other gluing theorems for a variety of other geometric structures. Gluing theorems are fundamental in gauge theory, and similar techniques have been developed and applied to metrics of constant scalar curvature [29], [26], minimal and constant mean curvature surfaces [21], [27], [28], and a variety of other geometric problems. Another recent result using the same circle of ideas, and relevant to this area of general relativity, is contained in [16]; this paper proves the existence of time-symmetric asymptotically flat solutions to the constraint equations (i.e. scalar flat asymptotically Euclidean metrics) which evolve to spacetimes which are identically Schwarzchild near spatial infinity. The technique common to many of these papers consists of a two-step procedure. First a one-parameter family of approximate solutions to any one of these problems is constructed. These are chosen so that the error measuring the discrepancy of these approximate solutions from exact solutions tends to zero, and so the strategy is to perturb these approximate solutions to exact solutions using a contraction mapping or implicit function theorem argument. 
The complication here is that as the parameter $\varepsilon$ converges to zero, the geometry is degenerating and some care must be taken to control the linearization of the nonlinear equation associated to the geometric problem. A slightly different and ultimately simpler approach is contained in [27], [28]; however, we shall follow the earlier and more familiar route here because in the present context either method requires approximately the same amount of work.

In the remainder of this section we set up some notation, give some definitions, state precise versions of our main results, and provide a more detailed guide to the rest of the paper.

\subsection{Statement of main results}

We fix a three-manifold $\Sigma$ along with a conformal class $[\gamma]$ on $\Sigma$. For any two points $p_{1}, p_{2}$ on this manifold, we refer to the triple $\left([\gamma], p_{1}, p_{2}\right)$ as a marked conformal structure on $\Sigma$. We have already alluded to a nondegeneracy condition required for the construction:

Definition 1 A marked conformal structure $\left([\gamma], p_{1}, p_{2}\right)$ on a compact manifold $\Sigma$ is nondegenerate if either of the following situations is true:

- $\Sigma$ is connected and there are no nontrivial conformal Killing vector fields on $\Sigma$ which vanish at either $p_{1}$ or $p_{2}$, or

- The points $p_{1}$ and $p_{2}$ lie on different components of $\Sigma$ and there are no conformal Killing fields which vanish at $p_{j}$ but which do not vanish identically on the component of $\Sigma$ containing $p_{j}$, for $j=1,2$.

Remark 1 To avoid trivialities, we always assume that either $\Sigma$ is connected or else has two components, each of which contains one of the points $p_{j}$. Note that this nondegeneracy condition is very mild and holds for any fixed conformal class $[\gamma]$ when the points $p_{j}$ are chosen generically. In the asymptotically Euclidean and asymptotically hyperboloidal cases, which are the only noncompact cases of interest to us here, this nondegeneracy condition is not needed. We discuss these cases in more detail in $\S 7$.

Assume that $\Sigma$ is compact, and fix two points $p_{1}, p_{2}$ on it. Suppose that $(\gamma, \Pi)$ is an initial data set with $\Pi \not \equiv 0$ and $\tau=\operatorname{tr}_{\gamma} \Pi$ constant, so that in particular the two constraint equations (11), (2) are satisfied. It is often more convenient to regard $(\gamma, \mu)$ as the initial data set instead, where $\mu$ is the transverse-traceless part of $\Pi$. For any (small) $R>0$, fix balls $B_{j}=B_{R}\left(p_{j}\right)$ of radius $R$ (with respect to $\gamma$ ) around the points $p_{j}$, and define

$$
\Sigma_{R}^{*}=\Sigma \backslash\left(B_{1} \cup B_{2}\right) .
$$

We also let $\Sigma_{0}^{*}=\Sigma^{*}=\Sigma \backslash\left\{p_{1}, p_{2}\right\}$. We shall modify the metric $\gamma$ conformally in each of the punctured balls $B_{j} \backslash\left\{p_{j}\right\}$ to obtain a metric $\gamma_{c}$ on $\Sigma^{*}$ with two asymptotically cylindrical ends. These ends have a natural parameter $t=-\log r$, where $r$ is the geodesic distance in $B_{j}$ to $p_{j}$. According to (5) the transverse-traceless tensor $\mu$ changes to a tensor $\mu_{c}$ which is transverse-traceless with respect to $\gamma_{c}$. This gives an asymptotically cylindrical pair $\left(\gamma_{c}, \mu_{c}\right)$ together with a function $\psi$ on $\Sigma^{*}$ which solves the associated 
Lichnerowicz equation (6) (with coefficients determined by this pair of tensors). We also refer to the triple $\left(\gamma_{c}, \mu_{c}, \psi\right)$ as an initial data set.

The construction proceeds as follows. We first identify long pieces, of length $T$, of these two cylindrical ends with one another to obtain a family of metrics $\gamma_{T}$ on the new manifold $\Sigma_{T}$ obtained from $\Sigma$ by adding a handle. For each $T, \Sigma_{T}$ is of course diffeomorphic to the manifold $\widehat{\Sigma}$ described in the previous section. This process involves cutoff functions and is not canonical. We also use cutoffs to patch together the values of $\mu_{c}$ on these ends to obtain $\mu_{T}$, and similarly an approximate solution $\psi_{T}$ of the Lichnerowicz equation. We write this out more carefully in $\$ 2$. Although it is not hard to construct $\mu_{T}$ so that it still has vanishing trace relative to $\gamma_{T}$, it is no longer necessarily true that $\mu_{T}$ will be divergence-free. The triple $\left(\gamma_{T}, \mu_{T}, \psi_{T}\right)$ does not solve the constraint equations, but instead gives an approximate solution, with an error term tending to zero as $T \rightarrow \infty$. We show that it is possible, for $T$ sufficiently large, to correct this data to obtain exact solutions of the constraint equations. We do this in the following order. First $\mu_{T}$ is altered by a small correction term $\sigma_{T}$, using a well known second order linear elliptic system (usually called the vector Laplacian) derived from the conformal Killing operator on $\left(\Sigma_{T}, \gamma_{T}\right)$, to obtain a tensor $\tilde{\mu}_{T}=\mu_{T}-\sigma_{T}$ which is transverse-traceless with respect to $\gamma_{T}$. This involves a careful analysis of the mapping properties of this operator with good control as $T \rightarrow \infty$ and is the topic of $\delta 3$. We then use the conformal method, and more specifically the Lichnerowicz equation (的), to modify the function $\psi_{T}$ to some $\tilde{\psi}_{T}$, so that $\left(\gamma_{T}, \tilde{\mu}_{T}, \tilde{\psi}_{T}\right)$ is an initial data set, i.e. gives an exact solution of the constraint equations. This involves first estimating the error terms for this approximate solution, which is done in $\S$. We next analyze the linearization of the Lichnerowicz equation uniformly as $T \rightarrow \infty$. In particular, we introduce a class of weighted Hölder spaces $\mathcal{C}_{\delta}^{k, \alpha}\left(\Sigma_{T}\right)$ which correspond to the standard Hölder spaces away from the handle and determine the range of weights $\delta$ for which the inverse of the linearized Lichnerowicz equation is uniformally bounded independent of $T$. This is carried out in $\$$. Finally, in $\$ 6$ we use a contraction mapping argument to produce the exact solutions by finding a function $\eta_{T}$ in the ball $\mathbb{B}_{\nu}$ of radius $\nu e^{-T / 4}$ in $\mathcal{C}_{\delta}^{k+2, \alpha}\left(\Sigma_{T}\right)$ for $\delta \in(0,1)$, so that $\tilde{\psi}_{T}=\psi_{T}+\eta_{T}$ satisfies the Lichnerowicz equation. This establishes our main theorem which we now state (in the form appropriate for initial data on closed manifolds).

Theorem 1 Let $\left(\Sigma, \gamma, \Pi ; p_{1}, p_{2}\right)$ be a compact, smooth, marked, constant mean curvature solution of the Einstein constraint equations; thus $\Pi=\mu+\frac{1}{3} \tau \gamma$ with $\tau$ constant and $\mu$ transverse-traceless with respect to $\gamma$. We assume that this solution is nondegenerate and also that $\Pi \not \equiv 0$. Then there is a geometrically natural choice of a parameter $T$ and, for $T$ sufficiently large, a one-parameter family of solutions $\left(\Sigma_{T}, \Gamma_{T}, \Pi_{T}\right)$ of the Einstein constraint equations with the following properties. First, the three-manifold $\Sigma_{T}$ is constructed from $\Sigma$ by adding a handle, or neck, connecting the two points $p_{1}$ and $p_{2}$. Next,

$$
\Gamma_{T}=\tilde{\psi}_{T}^{4} \gamma_{T} \quad \text { and } \quad \Pi_{T}=\tilde{\psi}_{T}^{-2} \tilde{\mu}_{T}+\frac{1}{3} \tau \tilde{\psi}_{T}^{4} \gamma_{T}
$$

where $\tilde{\psi}_{T}=\psi_{T}+\eta_{T}$ and $\tilde{\mu}_{T}=\mu-\sigma_{T}$ are very small perturbations of the conformal factor $\psi_{T}$ and approximate transverse-traceless tensor $\mu_{T}$ defined informally above and more carefully in $\S 2$ below. The tensor $\tilde{\mu}_{T}$ is transverse-traceless with respect to $\Gamma_{T}$ and the function $\tilde{\psi}_{T}$ satisfies the Lichnerowicz equation with respect to the pair $\left(\gamma_{T}, \tilde{\mu}_{T}\right)$. 
Finally, the perturbation terms satisfy the following estimates as $T \rightarrow \infty$ :

- $O n \Sigma_{R}^{*}$,

$$
\left|\Gamma_{T}-\gamma\right|_{\gamma} \leq C e^{-T / 4}, \quad\left|\Pi_{T}-\Pi\right|_{\gamma} \leq C T^{3} e^{-3 T / 2}
$$

- On the neck region, there is a natural choice of coordinate system $(s, \theta),-T / 2 \leq$ $s \leq T / 2$ and $\theta \in S^{2}$, such that if $h_{0}$ is the standard metric on $S^{2}$, then

$$
\begin{gathered}
\gamma_{T}=d s^{2}+h_{0}+\mathcal{O}\left(e^{-T / 2} \cosh s\right) \\
\psi_{T}=2 e^{-T / 4} \cosh (s / 2)+\mathcal{O}\left(e^{-T / 4}\right), \quad\left|\eta_{T}\right| \leq C e^{-(1+\delta) T / 4}(\cosh (s / 2))^{\delta}
\end{gathered}
$$

for some weight $\delta \in(0,1)$, and finally

$$
\left|\tilde{\mu}_{T}\right|_{\gamma_{T}} \leq C e^{-T / 2} \cosh s, \quad\left|\sigma_{T}\right|_{\gamma_{T}} \leq C T^{3} e^{-3 T / 2}
$$

The modifications needed for the asymptotically Euclidean and asymptotically hyperboloidal cases are discussed in $\delta[\sqrt{7}$ and statements of this theorem in these settings are given there, in Theorems 1 and 15 .

Notice that we have not stated the precise regularity of the solutions, but as indicated earlier, we work in various classes of Hölder spaces; in particular, we also obtain existence of initial data sets $(\Sigma, \gamma, \Pi)$ with only finite regularity.

Some estimates of the geometry of the solutions are provided in $\S 8$; these should be of interest when considering the spacetime evolutions of these initial data sets. Finally, $\S 9$ contains an informal discussion of various concrete examples of initial data sets, many of which are new, which our construction gives.

\section{The approximate solution}

In this section we construct the family of approximate solutions $\left(\gamma_{T}, \mu_{T}, \psi_{T}\right)$. Let $r_{j}$ denote the geodesic distance to $p_{j}$ (relative to $\gamma$ ) in the ball $B_{j}$. By Gauss' lemma we have

$$
\left.\gamma\right|_{B_{j}}=d r_{j}^{2}+r_{j}^{2} h_{j}\left(r_{j}\right)
$$

where $h_{j}$ is a family of metrics on $S^{2}$, smooth down to $r_{j}=0$ and such that $h_{j}(0)$ is the standard round metric on the sphere. The standard way to obtain a metric with asymptotically cylindrical ends is to multiply $\gamma$ by $r_{j}^{-2}$ in each of these balls. Recalling the convention of (俍) that the conformal factor is written as $\psi^{4}$, let us fix a function $\psi_{c}$ which is strictly positive on $\Sigma^{*}$, which equals one on $\Sigma_{2 R}^{*}$, and which equals $r_{j}^{1 / 2}$ on $B_{j}$. Now define

$$
\gamma_{c}=\psi_{c}^{-4} \gamma
$$

This metric agrees with $\gamma$ on $\Sigma_{2 R}^{*}$ and has asymptotically cylindrical ends in a neighborhood of each $p_{j}$. 

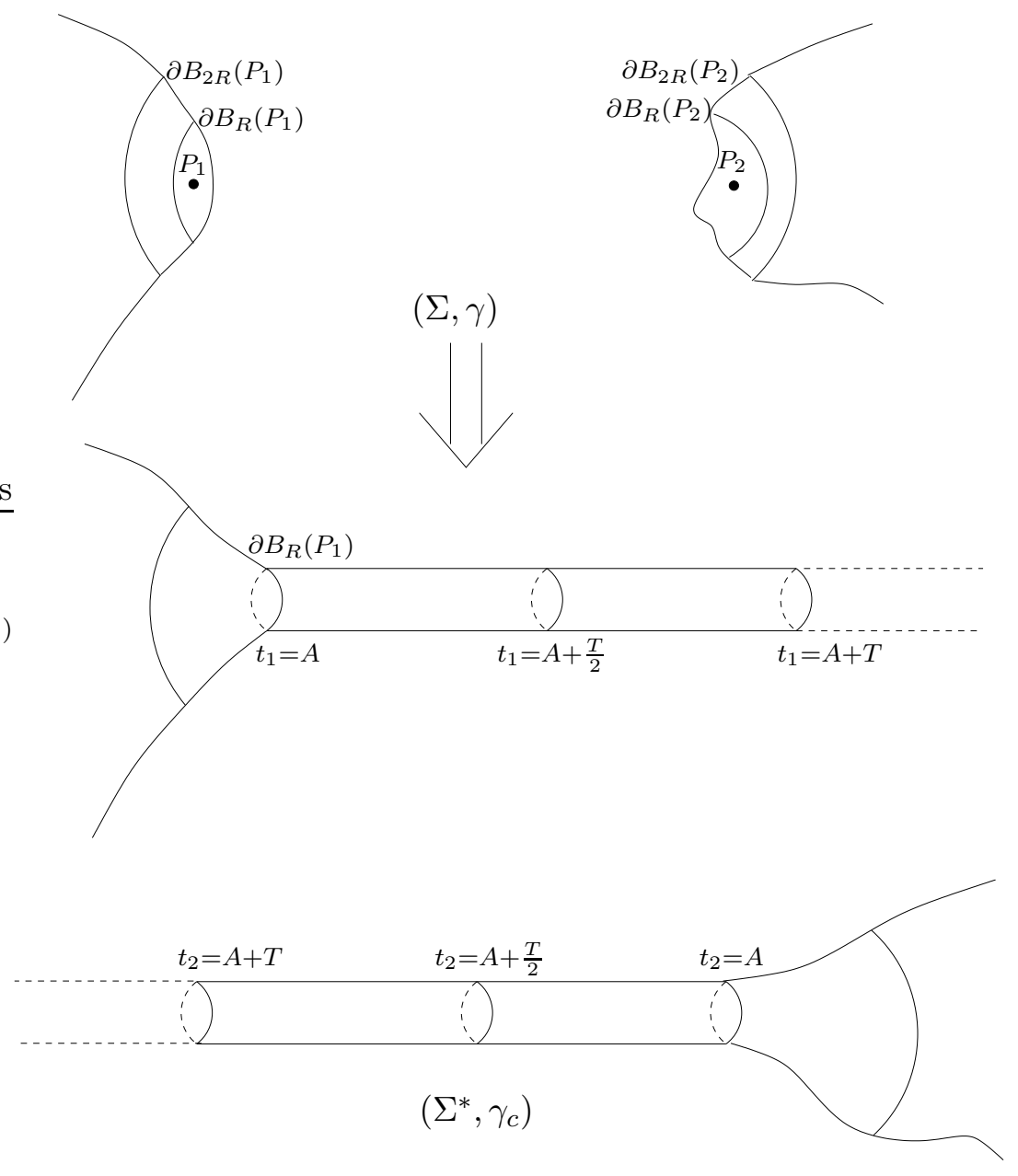

Figure 1: Conformally blowing up $(\Sigma, \gamma)$ to form $\left(\Sigma^{*}, \gamma_{c}\right)$ with two asymptotically cylindrical ends.

In fact, setting $t_{j}=-\log r_{j}$, we see that

$$
\left.\gamma_{c}\right|_{B_{j}}=d t_{j}^{2}+h_{j}\left(e^{-t_{j}}\right) .
$$

Recalling that $h_{j}$ is smooth in $r_{j}$, we can rewrite this as

$$
d t_{j}^{2}+d \theta^{2}+e^{-t_{j}} k_{j}
$$

where $d \theta^{2}$ is the standard round metric on $S^{2}$ and the term $k_{j}$ in the remainder is a symmetric two-tensor which is smooth in $r_{j}=e^{-t_{j}}$. Note that if the metric $\gamma$ is conformally flat in $B_{j}$ then the definition of $\psi_{c}$ may be easily modified to make the metric $\gamma_{c}$ exactly cylindrical in $B_{j}$. Note also, for later reference, that

$$
-\log R \leq t_{j}<\infty
$$

Next, following (5), define

$$
\mu_{c}=\psi_{c}^{2} \mu
$$


this is defined on $\Sigma^{*}$ and is transverse-traceless with respect to $\gamma_{c}$. Relative to the initial data set $\left(\gamma_{c}, \mu_{c}\right)$ on $\Sigma^{*}, \psi_{c}$ satisfies the Lichnerowicz equation

$$
\Delta_{c} \psi_{c}-\frac{1}{8} R_{c} \psi_{c}+\frac{1}{8}\left|\mu_{c}\right|^{2} \psi_{c}^{-7}-\frac{1}{12} \tau^{2} \psi_{c}^{5}=0
$$

In this equation, the expressions $\Delta_{c}, R_{c}$ and $|\cdot|^{2}$ are all computed in terms of the metric $\gamma_{c}$. Thus $\left(\gamma_{c}, \mu_{c}, \psi_{c}\right)$ is an initial data set on $\Sigma^{*}$.

Let $A=-\log R$ and let $T$ be a large parameter. Truncate the manifold $\Sigma^{*}$ by omitting the regions where $t_{j}>A+T$. We may form a new smooth manifold by identifying the two finite cylindrical tubes $\left\{\left(t_{j}, \theta\right): A \leq t_{j} \leq A+T\right\}$ via the map $\left(t_{1}, \theta\right) \rightarrow\left(T-t_{1},-\theta\right)$. Let us call the resulting manifold $\Sigma_{T}$ and let the now-identified cylindrical tube be denoted $C_{T}$. (Of course, each $\Sigma_{T}$ is diffeomorphic to $\widehat{\Sigma}$, the manifold obtained by adding a handle to $\Sigma$, but it is convenient to keep track of the dependence of $T$ explicitly.) We introduce a new linear coordinate $s$ on $C_{T}$ by setting

$$
s=t_{1}-A-T / 2=-t_{2}+A+T / 2 .
$$

Thus $C_{T}$ is parametrized by the coordinates $(s, \theta) \in[-T / 2, T / 2] \times S^{2}$.

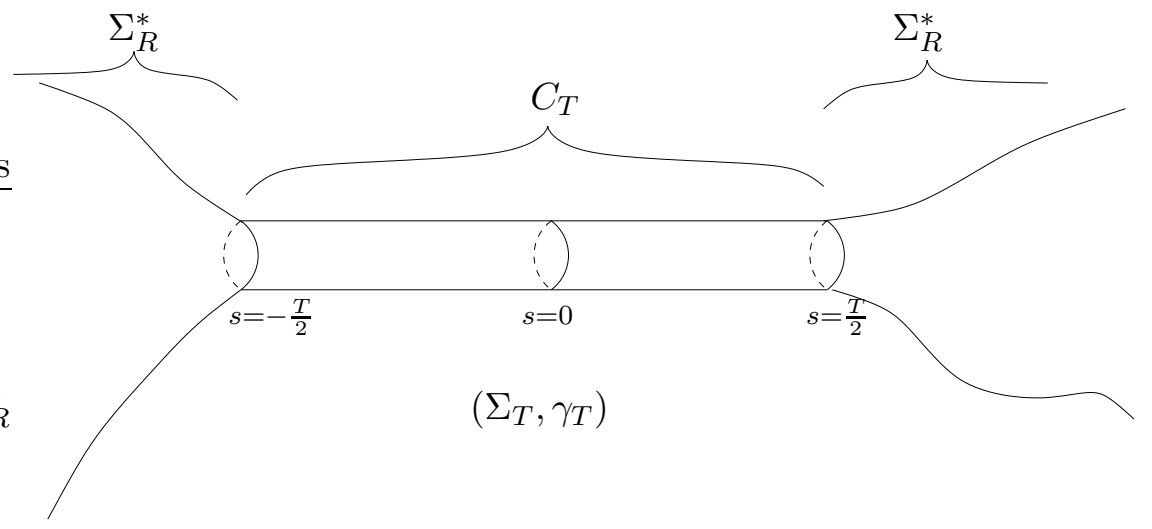

Figure 2: The manifold $\left(\Sigma_{T}, \gamma_{T}\right)$.

To continue we must define the family of metrics $\gamma_{T}$, 'almost-transverse-traceless' tensors $\mu_{T}$ on $\Sigma_{T}$, and conformal factors $\psi_{T}$, which we do in turn.

The definition of $\gamma_{T}$ is most transparent when $\gamma$ is conformally flat in the balls $B_{j}$. In this case, using the modifications of $\psi_{c}$ alluded to above, in the cylindrical coordinates $\left(t_{j}, \theta\right)$, we have $\gamma_{c}=d t_{j}^{2}+d \theta^{2}$, and thus the map identifying the two cylindrical pieces to $C_{T}$ is an isometry and $\gamma_{T}$ is thereby well-defined. In the general case, we cover $\Sigma_{T}$ by two open sets $\mathcal{U}_{1}$ and $\mathcal{U}_{2}$, the intersection of which consists of two components, one disjoint from $B_{1} \cup B_{2}$ and the other equal to $\{(s, \theta):-1<s<1\}$. Choose a partition of unity $\left\{\chi_{1}, \chi_{2}\right\}$ subordinate to this cover and let $\gamma_{j}$ and $\mu_{j}$ denote the restrictions of $\gamma_{c}$ and $\mu_{c}$ to $\mathcal{U}_{j}$. Then, with the obvious abuse of notation, define

$$
\gamma_{T}=\chi_{1} \gamma_{1}+\chi_{2} \gamma_{2}, \quad \mu_{T}=\chi_{1} \mu_{1}+\chi_{2} \mu_{2}
$$

Notice that $\gamma_{T}=\gamma_{c}$ and $\mu_{T}=\mu_{c}$ away from the middle $Q=[-1,1] \times S^{2}$ of the neck region, where $t_{1}, t_{2} \approx T / 2$. 


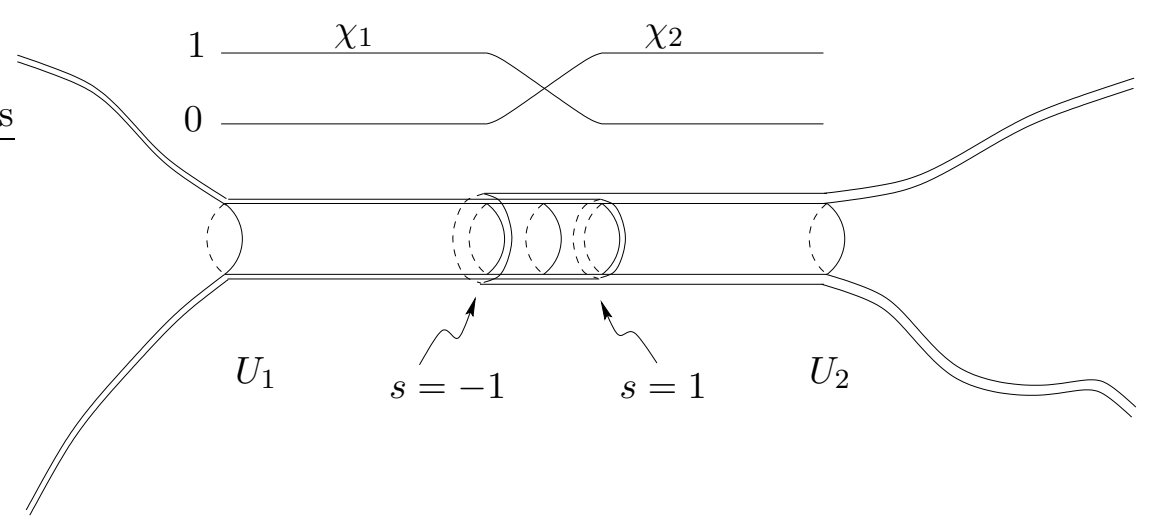

Figure 3: Defining the metric $\gamma_{T}=\chi_{1} \gamma_{1}+\chi_{2} \gamma_{2}$, and the approximate TT-tensor

$$
\mu_{T}=\chi_{1} \mu_{1}+\chi_{2} \mu_{2} \text {. }
$$

Although it is not obvious why at this stage, we need to define $\psi_{T}$ somewhat differently. This time choose a covering $\left\{\tilde{\mathcal{U}}_{1}, \tilde{\mathcal{U}}_{2}\right\}$ of $\Sigma$ such that $\tilde{\mathcal{U}}_{1} \cap \tilde{\mathcal{U}}_{2} \subset \Sigma_{2 R}^{*}$ and with $p_{j} \in \tilde{\mathcal{U}}_{j}$. Also choose nonnegative functions $\tilde{\chi}_{j} \in \mathcal{C}_{0}^{\infty}\left(\tilde{\mathcal{U}}_{j}\right)$ with $\tilde{\chi}_{1}+\tilde{\chi}_{2}=1$ on $\Sigma_{R}^{*}$, and such that when restricted to $B_{j}, \tilde{\chi}_{j}=1$ for $t_{j} \leq A+T-1$ and $\tilde{\chi}_{j}=0$ for $t_{j} \geq A+T$. Writing the restriction of $\psi_{c}$ to $\tilde{\mathcal{U}}_{j}$ as $\psi_{j}$, we set

$$
\psi_{T}=\tilde{\chi}_{1} \psi_{1}+\tilde{\chi}_{2} \psi_{2} .
$$

Then, based on the construction of $\Sigma_{T}$ from the conformal blow up of $(\Sigma, \gamma)$, we define $\psi_{T}$ on $\Sigma_{T}$ in the obvious way. Note that $\psi_{T}$ is exactly equal to 1 away from the cylinder, and is equal to $\psi_{1}+\psi_{2}$ throughout most of the cylinder except at the two ends. At these ends, near the junctions of the cylinder with $\Sigma^{R}$, one of the two functions $\psi_{1}$ or $\psi_{2}$ is roughly of unit size and the other is exponentially small; in defining $\psi_{T}$, the exponentially small summand is cut off to be zero near these junctures.

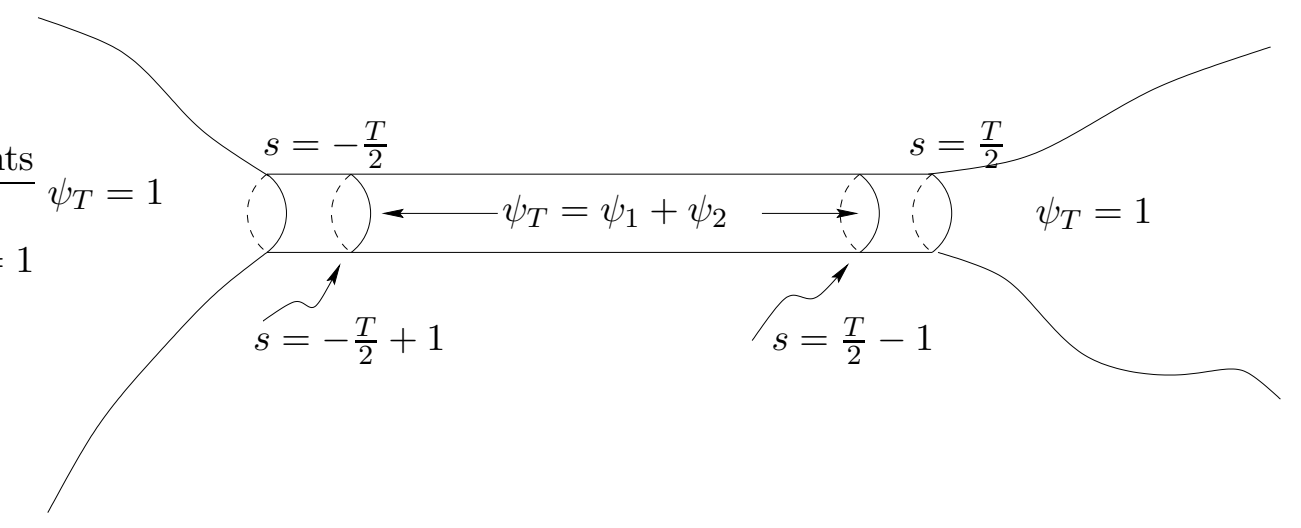

Figure 4: The approximate solution to the Lichnerowicz equation, $\psi_{T}=\tilde{\chi}_{1} \psi_{1}+\tilde{\chi}_{2} \psi_{2}$.

In summary, we have defined $\gamma_{T}$ and $\mu_{T}$ by using cutoffs supported on the middle of the cylinder $C_{T}$, but have cut off either of the $\psi_{j}$ at the ends of the cylinder. The reasons for doing this are that $\gamma_{c}$ decays to the product metric like $e^{-t_{j}}$ and $\mu_{c}$ is extremely small in the middle of $C_{T}$, so the error terms from these are of order $e^{-T / 2}$. On the other 
hand, $\psi_{c}$ only decays like $e^{-t_{j} / 2}$ along each end, and so if we were to cut this function off in the middle, it would produce an error term with size approximately $e^{-T / 4}$, which is unacceptably large. We carry out these estimates in more detail in $\S$.

\section{Transverse-traceless tensors on $\Sigma_{T}$}

We now undertake the first of several steps intended to perturb the approximate solution $\left(\gamma_{T}, \mu_{T}, \psi_{T}\right)$ constructed in the last section to an initial data set, i.e. an exact solution, when $T$ is sufficiently large. Our goal in this section is to modify the tensor $\mu_{T}$ so that it becomes transverse-traceless with respect to $\gamma_{T}$.

Before starting, note that it is clear from the definitions that $\mu_{T}$ is transverse-traceless away from the center of the neck region $Q$ in the middle of the cylinder $C_{T}$. Furthermore, by choosing the cutoff functions carefully, we may also assume that $\mu_{T}$ is trace-free on all of $\Sigma_{T}$; however, its divergence is almost surely nonzero, but at least is supported in the annulus $Q$.

There is a well-known procedure for producing a correction term to kill this divergence. We recall this now, and refer to [15] or [33] for more details. Let $\gamma$ be a metric and $X$ a vector field on the three-manifold $\Sigma$, and write

$$
\mathcal{D} X=\frac{1}{2} \mathcal{L}_{X} \gamma-\frac{1}{3}(\operatorname{div} X) \gamma
$$

Equivalently, in local coordinates,

$$
(\mathcal{D} X)_{j k}=\frac{1}{2}\left(X_{j ; k}+X_{k ; j}\right)-\frac{1}{3} \operatorname{div}(X) \gamma_{j k} .
$$

The first order operator $\mathcal{D}=\mathcal{D}_{\gamma}$ defined by this expression maps vector fields to tracefree symmetric $(0,2)$ tensors and has the property that $\mathcal{D} X=0$ if and only if $X$ is a conformal Killing field. The formal adjoint of $\mathcal{D}$ is $\mathcal{D}^{*}=-\operatorname{div}$, and the second order operator $L=\mathcal{D}^{*} \circ \mathcal{D}$ is self-adjoint, nonnegative and elliptic. This operator is often referred to as the 'vector Laplacian'.

Now let $\gamma=\gamma_{T}$, with $\mathcal{D}$ and $L$ the associated operators. Let $W$ be the vector field associated to div $\mu_{T}$ using the natural duality between vector fields and 1-forms. Suppose that we can solve the equation $L X=W$. Then writing $\sigma_{T}=\mathcal{D} X$ we compute that

$$
\operatorname{div}\left(\mu_{T}-\sigma_{T}\right)=W-\operatorname{div} \mathcal{D} X=W-L X=0,
$$

and so $\mu_{T}-\sigma_{T}$ is divergence-free. Since $\mu_{T}$ and $\sigma_{T}$ are both trace-free, we have produced the desired correction term.

To implement this strategy properly, we must show that when $T$ is large enough, the operator $L$ is invertible, and moreover that the solution $X$, and hence $\mathcal{D} X$, is much smaller than $\mu_{T}$, so that $\sigma_{T}$ can honestly be regarded as a small perturbation. Note that since $W$ is a divergence and hence orthogonal to the cokernel of $L$, we can always solve $L X=W$ (but without an estimate on the solution). The main issue is to prove that the inverse $G$ to $L$ on the manifold $\Sigma_{T}$, when acting between appropriate function spaces, has norm bounded at worst by some polynomial in $T$. (This is sufficient because the error term $\operatorname{div} \mu_{T}$ decays exponentially.) 
When $\gamma$ is conformally flat in the balls $B_{j}$, it is possible to choose the conformal factor $\psi$ used to define $\gamma_{c}$ slightly differently so that $\gamma_{T}$ is the product metric on the cylinder $C_{T}=[-T / 2, T / 2] \times S^{2}$. We proceed in this case by an analysis of the operators $\mathcal{D}$ and $L$ on the complete cylinder $\mathbb{R} \times S^{2}$ as well as on the finite cylindrical piece $C_{T}$, using separation of variables and explicit calculations with the resulting ODEs, to reach the conclusion that the inverse $G$ to $L$ exists and has norm (as an operator on $L^{2}\left(\Sigma_{T}\right)$ ) blowing up no faster than $T^{2}$. When $\gamma$ is not conformally flat in these balls, an additional perturbation argument is required to reach the same conclusion. The basic goal in these arguments is to show that the lowest Dirichlet eigenvalue of $L$ on $C_{T}$ is of order $T^{-2}$. Afterwards, the same conclusion is reached for the operator $L$ on $\Sigma_{T}$.

\subsection{The conformal Killing operator on the cylinder}

\subsubsection{The decomposition}

We first derive the explicit form of $L$ on the cylinder $\mathbb{R} \times S^{2}$ with product metric $d s^{2}+h$, where $s$ is the linear coordinate on $\mathbb{R}$ and $h$ is the standard round metric on $S^{2}$. Any vector field $X$ on the cylinder may be written

$$
X=f \partial_{s}+Y(s)
$$

where $f$ is a function on the cylinder and $Y(s)$ is a vector field on the sphere $\{s\} \times S^{2}$. Write $\mathcal{D} X=S(X)-\frac{1}{3} \operatorname{div} X \gamma$, and choose any local coordinates $\theta=\left(\theta_{1}, \theta_{2}\right)$ on $\mathbb{S}^{2}$. Then for $i, j=1,2$,

$$
S\left(f \partial_{s}\right)_{00}=\partial_{s} f, \quad S\left(f \partial_{s}\right)_{0 i}=\frac{1}{2} f_{i}, \quad S\left(f \partial_{s}\right)_{i j}=0
$$

and $\operatorname{div}\left(f \partial_{s}\right)=\partial_{s} f$. Therefore, exhibited as a matrix,

$$
\mathcal{D}\left(f \partial_{s}\right)=\left(\begin{array}{cc}
\frac{2}{3} \partial_{s} f & \frac{1}{2} \nabla_{\theta} f \\
\frac{1}{2} \nabla_{\theta} f & -\frac{1}{3}\left(\partial_{s} f\right) h
\end{array}\right)
$$

where $\nabla_{\theta}$ represents the gradient of $f$ on the sphere. Hence, with the convention $\Delta_{\theta}=$ $-\operatorname{div} \nabla_{\theta}$

$$
L\left(f \partial_{s}\right)=-\operatorname{div}\left(\mathcal{D}\left(f \partial_{s}\right)\right)=\left(\begin{array}{c}
-\frac{2}{3} \partial_{s}^{2} f+\frac{1}{2} \Delta_{\theta} f \\
-\frac{1}{2} \partial_{s}\left(\nabla_{\theta} f\right)+\frac{1}{3} \nabla_{\theta}\left(\partial_{s} f\right)
\end{array}\right)=\left(\begin{array}{c}
-\frac{2}{3} \partial_{s}^{2} f+\frac{1}{2} \Delta_{\theta} f \\
-\frac{1}{6} \partial_{s}\left(\nabla_{\theta} f\right)
\end{array}\right) .
$$

Next,

$$
S(Y)_{00}=0, \quad S(Y)_{0 i}=\frac{1}{2} \partial_{s}\left(Y_{i}\right), \quad S(Y)_{i j}=S_{\theta}(Y)_{i j}
$$

where by definition the conformal Killing operator $\mathcal{D}_{h}$ on the sphere decomposes as $\mathcal{D}_{h} Y=S_{\theta}(Y)-\frac{1}{2}\left(\operatorname{div}_{\theta} Y\right) h$. Using also that $\operatorname{div}(Y)=\operatorname{div}_{\theta}(Y)$, we get

$$
\mathcal{D}(Y)=\left(\begin{array}{cc}
-\frac{1}{3} \operatorname{div}_{\theta}(Y) & \frac{1}{2} \partial_{s} Y \\
\frac{1}{2} \partial_{s} Y & \mathcal{D}_{\theta}(Y)+\frac{1}{6} \operatorname{div}_{\theta}(Y) h
\end{array}\right),
$$


and then that

$$
\begin{gathered}
L(Y)=-\operatorname{div}(\mathcal{D}(Y))=\left(\begin{array}{c}
\frac{1}{3} \partial_{s}\left(\operatorname{div}_{\theta}(Y)\right)-\frac{1}{2} \operatorname{div}_{\theta}\left(\partial_{s} Y\right) \\
-\frac{1}{2} \partial_{s}^{2} Y-\operatorname{div}_{\theta}\left(\mathcal{D}_{\theta}(Y)\right)-\frac{1}{6} \nabla_{\theta} \operatorname{div}_{\theta}(Y)
\end{array}\right) \\
=\left(\begin{array}{c}
-\frac{1}{6} \partial_{s}\left(\operatorname{div}_{\theta}(Y)\right) \\
-\frac{1}{2} \partial_{s}^{2} Y+L_{\theta}(Y)-\frac{1}{6} \nabla_{\theta} \operatorname{div}_{\theta}(Y)
\end{array}\right) .
\end{gathered}
$$

It is possible to express this operator in a somewhat more familiar form. To do this, we identify vector fields and 1-forms on $S^{2}$ in the usual way using the metric $h$. Thus, $\nabla_{\theta}$ and $\operatorname{div}_{\theta}$ correspond to $d_{\theta}$ and $-\delta_{\theta}$, respectively. We still let $Y$ denote the 1 -form associated to the vector field $Y$, hopefully without causing undue confusion. Using these identifications,

$$
L(f d s+Y(s))=\left(\begin{array}{c}
-\frac{2}{3} \partial_{s}^{2} f+\frac{1}{2} \Delta_{\theta} f+\frac{1}{6} \partial_{s}\left(\delta_{\theta} Y\right) \\
-\frac{1}{2} \partial_{s}^{2} Y+L_{\theta}(Y)+\frac{1}{6} d_{\theta} \delta_{\theta}(Y)-\frac{1}{6} \partial_{s}\left(d_{\theta} f\right)
\end{array}\right) .
$$

This expression may be simplified using two separate Weitzenböck formulæ,

$$
L_{\theta}=\frac{1}{2} \nabla^{*} \nabla-\frac{1}{2}, \quad \text { and } \quad \Delta_{\theta}=\nabla^{*} \nabla+1,
$$

both of which use the fact that the curvature tensor on $S^{2}$ is constant. The first of these relates $L_{\theta}$ to the 'rough Laplacian' $\nabla_{\theta}^{*} \nabla_{\theta}$ (cf. [33]) and the second is the more familiar one relating this rough Laplacian to the Hodge Laplacian $\Delta_{\theta}=d_{\theta} \delta_{\theta}+\delta_{\theta} d_{\theta}$. These combine to give

$$
L_{\theta}=\frac{1}{2} \Delta_{\theta}-1
$$

and thus we finally arrive at the expression

$$
L\left(\begin{array}{c}
f \\
Y
\end{array}\right)=\left(\begin{array}{cc}
-\frac{2}{3} \partial_{s}^{2}+\frac{1}{2} \Delta_{\theta} & \frac{1}{6} \partial_{s} \delta_{\theta} \\
-\frac{1}{6} \partial_{s} d_{\theta} & -\frac{1}{2} \partial_{s}^{2}+\frac{2}{3} d_{\theta} \delta_{\theta}+\frac{1}{2} \delta_{\theta} d_{\theta}-1
\end{array}\right)\left(\begin{array}{c}
f \\
Y
\end{array}\right) .
$$

\subsubsection{Separation of variables}

To analyze this operator $L$ on the cylinder in more detail, we introduce the eigenfunction expansion on the $S^{2}$ factor. Let $\left\{\phi_{k}\right\}$ be an orthonormal set of eigenfunctions for the scalar Laplacian on $S^{2}$, so that $\Delta_{\theta} \phi_{k}=\lambda_{k} \phi_{k}$ and each $\lambda_{k}=j(j+1)$ for some $j \in \mathbb{N}$. If $*$ is the Hodge star on $S^{2}$, then $\left\{\lambda_{k}^{-1 / 2} d_{\theta} \phi_{k}, \lambda_{k}^{-1 / 2} \delta_{\theta} * \phi_{k}\right\}$ is an orthonormal basis of eigenfunctions for the Hodge Laplacian on 1-forms. Set $\phi=\phi_{k}$ and $\lambda=\lambda_{k}$ with $\lambda_{k}>0$, for some $k$, and consider a 1 -form of the form

$$
X=f(s, \theta) d s+Y(s)=u(s) \phi d s+v(s) \frac{d_{\theta} \phi}{\sqrt{\lambda}}+w(s) \frac{\delta_{\theta} * \phi}{\sqrt{\lambda}},
$$


or equivalently, the associated dual vector field. Then

$$
\begin{gathered}
L X=\left(-\frac{2}{3} u^{\prime \prime}+\frac{\lambda}{2} u+\frac{\sqrt{\lambda}}{6} v^{\prime}\right) \phi d s+\left(-\frac{1}{2} v^{\prime \prime}+\left(\frac{2}{3} \lambda-1\right) v-\frac{\sqrt{\lambda}}{6} u^{\prime}\right) \frac{d_{\theta} \phi}{\sqrt{\lambda}} \\
+\left(-\frac{1}{2} w^{\prime \prime}+\left(\frac{1}{2} \lambda-1\right) w\right) \frac{\delta_{\theta} * \phi}{\sqrt{\lambda}},
\end{gathered}
$$

and so $L$ acts on the column vector $(u, v, w)^{t}$ by

$$
\left(\begin{array}{ccc}
-\frac{2}{3} & 0 & 0 \\
0 & -\frac{1}{2} & 0 \\
0 & 0 & -\frac{1}{2}
\end{array}\right) \partial_{s}^{2}+\left(\begin{array}{ccc}
0 & \frac{\sqrt{\lambda}}{6} & 0 \\
-\frac{\sqrt{\lambda}}{6} & 0 & 0 \\
0 & 0 & 0
\end{array}\right) \partial_{s}+\left(\begin{array}{ccc}
\frac{\lambda}{2} & 0 & 0 \\
0 & \frac{2}{3} \lambda-1 & 0 \\
0 & 0 & \frac{1}{2} \lambda-1
\end{array}\right)
$$

There is one extra case, when $\lambda=\lambda_{0}=0$; then $X=u(s) d s$ and $L X=-\frac{2}{3} u^{\prime \prime}(s) d s$, so we write

$$
L_{0}=-\frac{2}{3} \partial_{s}^{2}
$$

When $j \geq 1, L_{j}$ uncouples as $L_{j}=L_{j}^{\prime} \oplus L_{j}^{\prime \prime}$ where

$$
L_{j}^{\prime}=\left(\begin{array}{cc}
-\frac{2}{3} & 0 \\
0 & -\frac{1}{2}
\end{array}\right) \partial_{s}^{2}+\left(\begin{array}{cc}
0 & \frac{\sqrt{\lambda}}{6} \\
-\frac{\sqrt{\lambda}}{6} & 0
\end{array}\right) \partial_{s}+\left(\begin{array}{cc}
\frac{\lambda}{2} & 0 \\
0 & \frac{2}{3} \lambda-1
\end{array}\right)
$$

acts on $(u, v)^{t}$ and

$$
L_{j}^{\prime \prime}=-\frac{1}{2} \partial_{s}^{2}+\left(\frac{1}{2} \lambda-1\right)
$$

acts on $w$. Thus altogether,

$$
L=L_{0} \oplus \bigoplus_{j \geq 1}\left(L_{j}^{\prime} \oplus L_{j}^{\prime \prime}\right)
$$

\subsubsection{Temperate solutions}

The first application of these formulæ concerns the solutions of $L X=0$ on the cylinder which do not grow exponentially in either direction.

Proposition 1 The space of temperate solutions to $L X=0$ on the cylinder $\mathbb{R} \times S^{2}$ is 8-dimensional and is spanned by the 2-dimensional family of $\theta$-independent radial vector fields $(a+b s) \partial_{s}, a, b \in \mathbb{R}$, and the 6-dimensional family of vector fields which are dual to the 1 -forms $\left(c_{i}+d_{i} s\right) \delta_{\theta} * \phi_{i}, c_{i}, d_{i} \in \mathbb{R}$, where $\phi_{i}=\left.x_{i}\right|_{S^{2}}, i=1,2,3$.

Proof: It is straightforward to check that these bounded or linearly growing vector fields are all in the nullspace of $L$. In fact, using the eigendecomposition of $L$ above, $L_{0} u=-\frac{2}{3} u^{\prime \prime}=0$ implies $u(s)=a+b s$, and this gives the first family. Next, for $j=1$ (i.e. $\lambda=2$ ), $L_{1}^{\prime \prime} w=-\frac{1}{2} w^{\prime \prime}=0$ implies $w(s)=c+d s$; the eigenfunctions here are the restrictions of linear functions on $\mathbb{R}^{3}$ to $S^{2}$, and so this gives the other family. It remains to show that these are the only temperate solutions. 
It is clear that when $j \geq 2$, hence $\lambda \geq 6$, all solutions of $L_{j}^{\prime \prime} w=0$ grow exponentially in one direction or the other. On the other hand, consider the homogeneous system $L_{j}^{\prime}$ when $j \geq 1$, which is

$$
\begin{aligned}
\frac{2}{3} u^{\prime \prime}-\frac{\lambda}{2} u-\frac{\sqrt{\lambda}}{6} v^{\prime} & =0 \\
\frac{1}{2} v^{\prime \prime}-\left(\frac{2}{3} \lambda-1\right) v+\frac{\sqrt{\lambda}}{6} u^{\prime} & =0,
\end{aligned}
$$

where $\lambda=j(j+1)$. This is a constant coefficient system, and solutions have the form

$$
\left(\begin{array}{l}
u \\
v
\end{array}\right)=e^{\rho s} z_{1}, \quad \text { or } \quad s e^{\rho s} z_{1}+e^{\rho s} z_{2}, \quad z_{1}, z_{2} \in \mathbb{C}^{2},
$$

where $\rho$ is a root of the indicial equation

$$
\operatorname{det}\left(\begin{array}{cc}
\frac{2}{3} \rho^{2}-\frac{\lambda}{2} & -\frac{\sqrt{\lambda}}{6} \rho \\
\frac{\sqrt{\lambda}}{6} \rho & \frac{1}{2} \rho^{2}-\left(\frac{2}{3} \lambda-1\right)
\end{array}\right)=\frac{1}{3}\left(\rho^{4}+2(1-\lambda) \rho^{2}+\left(\lambda^{2}-\frac{3}{2} \lambda\right)\right)=0 .
$$

Solutions are temperate only when $\operatorname{Re} \rho=0$, but it is easy to check that this polynomial for $\rho$ has no root with purely imaginary part, since $\lambda=j(j+1), j \geq 1$. The proof is complete.

Remark 2 The space of conformal Killing fields on $S^{3}$ is 10-dimensional, and is generated by the 6-dimensional family of rotations and the 4-dimensional family of dilations. Amongst all of these, only the vector fields vanishing at the two antipodal points (corresponding to the infinite ends on the cylinder) give rise to temperate conformal Killing fields on $\mathbb{R} \times S^{2}$. Of course, these are just the generators for the 3-dimensional family of rotations of the orthogonal $S^{2}$ (i.e. corresponding to $\delta_{\theta} * \phi_{i}, i=1,2,3$, as above) and the dilation fixing these antipodal points $\left(\partial_{s}\right)$.

\subsubsection{The lowest eigenvalue of $L$ on $[-T / 2, T / 2] \times S^{2}$}

The next application concerns the lowest eigenvalue of the operator $L$ with Dirichlet boundary conditions $X=0$ on the finite piece of the cylinder $C_{T}=[-T / 2, T / 2] \times S^{2}$.

Proposition 2 Let $\lambda_{0}=\lambda_{0}(T)$ denote the first Dirichlet eigenvalue for $L$ on $C_{T}=$ $[-T / 2, T / 2] \times S^{2}$. Then

$$
\lambda_{0}(T) \geq \frac{C}{T^{2}}
$$

for some constant $C$ independent of $T$.

Proof: This estimate is clearly sharp for the scalar ordinary differential operators $L_{0}=$ $-\frac{2}{3} \partial_{s}^{2}$ and $L_{1}^{\prime \prime}=-\frac{1}{2} \partial_{s}^{2}$. On the other hand, when $j \geq 2$, the lowest eigenvalue of $L_{j}^{\prime \prime}$ converges to $\frac{1}{2} j(j+1)-1$ as $T \rightarrow \infty$, hence is bounded away from zero. 
We finally show that the lowest eigenvalue for $L_{j}^{\prime}$ is also bounded away from zero when $j \geq 1$. In fact,

$$
\begin{gathered}
\left\langle L_{j}^{\prime}\left(\begin{array}{l}
u \\
v
\end{array}\right),\left(\begin{array}{c}
u \\
v
\end{array}\right)\right\rangle=\int_{-T / 2}^{T / 2} \frac{2}{3}\left(u^{\prime}\right)^{2}+\frac{1}{2}\left(v^{\prime}\right)^{2}+\frac{\sqrt{\lambda}}{6}\left(u v^{\prime}-u^{\prime} v\right)+\frac{\lambda}{2} u^{2}+\left(\frac{2}{3} \lambda-1\right) v^{2} d s \\
=\int_{-T / 2}^{T / 2} \frac{2}{3}\left(u^{\prime}-\frac{\sqrt{\lambda}}{8} v\right)^{2}+\frac{1}{2}\left(v^{\prime}+\frac{\sqrt{\lambda}}{6} u\right)^{2}+\frac{35}{72} \lambda u^{2}+\left(\frac{63}{96} \lambda-1\right) v^{2} d s .
\end{gathered}
$$

Since $\lambda \geq 2$, this is bounded below by $\frac{1}{2} \int_{0}^{T}\left(u^{2}+v^{2}\right) d s$, and the proposition is proved.

\subsubsection{Growth estimates on $C_{T}$}

To conclude this analysis of $L$ on the cylinder, we show that if $X$ is an eigenfunction with 'very small' eigenvalue, then its size in the interior of $C_{T}$ is controlled by its size on the ends of $C_{T}$. The next result does this directly for the product metric. After that we prove a more technical estimate for the inhomogeneous eigenvalue problem which will be used later to handle the general case by perturbation.

Proposition 3 Suppose that $L X=\mu X$, where $L$ is computed with respect to the product metric and $\mu<\frac{1}{8} \lambda_{0}(T)$. Suppose also that

$$
\int_{S^{2}}\left(|X(-T / 2, \theta)|^{2}+|X(T / 2, \theta)|^{2}\right) d \theta \leq C_{1} .
$$

Then for any $a \in[-T / 2+1, T / 2-1]$,

$$
\int_{a-1}^{a+1} \int_{S^{2}}|X(s, \theta)|^{2} d s d \theta \leq C_{2}
$$

where $C_{2}$ depends only on $C_{1}$, but not on $T$ or $a$, and where $C_{2} \rightarrow 0$ as $C_{1} \rightarrow 0$.

Proof: It suffices to check this estimate on each of the components in the decomposition of $L$. For the scalar operators $L_{0}$ and $L_{j}^{\prime \prime}$ this estimate may be verified using the explicit solutions of the equation (though this can easily be done by other standard and less computational methods too). So as before, it suffices to focus on the system $L_{j}^{\prime}, j \geq 1$. Write

$$
L_{j}^{\prime}=-A \partial_{s}^{2}+B \partial_{s}+C
$$

where

$$
A=\left(\begin{array}{cc}
2 / 3 & 0 \\
0 & 1 / 2
\end{array}\right), \quad B=\left(\begin{array}{cc}
0 & \sqrt{\lambda} / 6 \\
-\sqrt{\lambda} / 6 & 0
\end{array}\right), \quad C=\left(\begin{array}{cc}
\lambda / 2 & 0 \\
0 & ((2 / 3) \lambda-1)
\end{array}\right),
$$

and, as usual, $\lambda=j(j+1)$.

Now write $X=(u, v)^{t}$ and define $f(s)=\langle A X, X\rangle$. Then

$$
\frac{1}{2} \partial_{s}^{2} f(s)=\left\langle A X^{\prime \prime}, X\right\rangle+\left\langle A X^{\prime}, X^{\prime}\right\rangle
$$


Using the equation $L_{j}^{\prime} X=\mu X$ and the skew-symmetry of $B$, this becomes

$$
\frac{1}{2} \partial_{s}^{2} f(s)=-\left\langle X^{\prime}, B X\right\rangle+\langle C X, X\rangle+\left\langle A X^{\prime}, X^{\prime}\right\rangle-\mu\langle X, X\rangle,
$$

which can then be rewritten as

$$
\frac{1}{2} \partial_{s}^{2} f(s)=\left|A^{1 / 2} X^{\prime}-\frac{1}{2} A^{-1 / 2} B X\right|^{2}+\langle D X, X\rangle,
$$

where

$$
D=\frac{1}{4} B A^{-1} B+C-\mu I=\left(\begin{array}{cc}
\frac{35}{72} \lambda-\mu & 0 \\
0 & \frac{63}{96} \lambda-1-\mu
\end{array}\right) .
$$

But $D$ is positive definite when $\lambda \geq 2$ and $T$ is large enough that subtracting $\mu$ does not destroy positivity, and so we conclude that $f^{\prime \prime}(s) \geq 0$, and hence that the $L^{2}$ norm of $X$ at $s= \pm T / 2$ controls the $L^{2}$ norm of $X$ over any strip $a-1 \leq s \leq a+1$.

Our second result here is more specialized, and is the precise additional ingredient required later to handle the nonproduct case. We let $\|\cdot\|_{0, \alpha, \sigma}$ denote the $\mathcal{C}^{0, \alpha}$ norm over the set $[\sigma, \sigma+1] \times S^{2}$.

Proposition 4 Let $L$ be computed relative to the product metric on $C_{T}$, and suppose $L X=\mu X+F$, where $X( \pm T / 2)=0$ and $\mu T^{2}<\nu_{0}$ for some sufficiently small constant $\nu_{0}$ independent of $T$. Suppose also that for all $|s| \leq T / 2$,

$$
\|F(s, \theta)\|_{0, \alpha, \sigma} \leq f(\sigma) e^{-T / 2} \cosh \sigma,
$$

where $|f(\sigma)| \leq 1$ for all $\sigma$. Recalling that $F$ implicitly depends on $T$, we also assume that for any $\varepsilon>0$ and $A>0$, there exists a $T_{0}$ such that when $T \geq T_{0}$ we have $|f(\sigma)| \leq \varepsilon$ when $T / 2-A \leq|\sigma| \leq T / 2$. Then, for any $\eta>0$ we have $|X| \leq \eta$ on all of $C_{T}$, when $T$ is large enough.

In other words, if $X$ solves this inhomogeneous eigenvalue problem on $C_{T}$, with vanishing Dirichlet data, if $\mu$ is much smaller than $1 / T^{2}$, and if the forcing term $F$ is uniformly small near the ends of $C_{T}$ and bounded by $e^{-T / 2} \cosh s$ on the middle portion of this cylinder, uniformly as $T \rightarrow \infty$, then $X$ is uniformly small on all of $C_{T}$.

Proof: We separate variables and write the equation as $L_{j} X_{j}=F_{j}$ for $j \geq 0$. For $j>0$ this equation splits further into $L_{j}^{\prime} X_{j}^{\prime}=F_{j}^{\prime}$ and $L_{j}^{\prime \prime} X_{j}^{\prime \prime}=F_{j}^{\prime \prime}$, as in the previous subsection. None of the primes here correspond to derivatives.

We have shown that the operators $L_{j}^{\prime}$ with $j \geq 1$ or $L_{j}^{\prime \prime}$ with $j \geq 2$ have spectrum bounded below by a positive constant as $T \rightarrow \infty$. For any of the corresponding equations, if the conclusion were false then we would be able to produce, by the usual arguments, a nontrivial bounded function $X^{\prime}$ or $X^{\prime \prime}$ such that $L_{j}^{\prime} X^{\prime}=0$ or $L_{j}^{\prime \prime} X^{\prime \prime}=0$, respectively, defined on the complete cylinder $\mathbb{R} \times S^{2}$. This contradicts the strictly positive lower bound for the spectrum.

For the remaining components, it suffices to prove the assertion for the scalar problem $\partial_{s}^{2} u+\alpha^{2} u=F$, where $\alpha T$ is sufficiently small. For notational convenience we shall use 
the shifted variable $t=s+T / 2$ (so that $F(t)=f(t) e^{-T / 2} \cosh (t-T / 2$ ), where this function $f$ is a translate of the one above. We may write the solution explicitly:

$$
u(t)=\frac{\sin \alpha(T-t)}{\sin \alpha T} \int_{0}^{t} \frac{\sin \alpha \tau}{\alpha T} F(\tau) d \tau+\frac{\sin \alpha t}{\alpha T} \int_{t}^{T} \frac{\sin \alpha(T-\tau)}{\sin \alpha T} F(\tau) d \tau .
$$

Because $\alpha T$ is so small, we may approximate each of the sines with their first order Taylor approximations; using the estimate for $F$ too we obtain

$$
\begin{aligned}
|u(t)| \leq C\left(\left(1-\frac{t}{T}\right)\right. & \int_{0}^{t} \tau \cosh (\tau-T / 2) e^{-T / 2} f(\tau) d \tau \\
& \left.+t \int_{t}^{T}\left(1-\frac{\tau}{T}\right) \cosh (\tau-T / 2) e^{-T / 2} f(\tau) d \tau\right) .
\end{aligned}
$$

Finally, we must use the separate upper bounds for $f(\tau)$ in the various regions $0 \leq \tau \leq A$, $A \leq \tau \leq T-A$ and $T-A \leq \tau \leq T$. The main fact used in this estimation is that the function $h(t)=t(1-t / T)|\sinh (t-T / 2)| e^{-T / 2}$ is uniformly bounded independently of $T$, attains its maximum near $t=1$ and $t=T-1$, and for any $\delta>0$ there exists an $A>0$ such that $h(t) \leq \delta$, uniformly in $T$, when $A \leq t \leq T-A$. Together with the bounds for $f$, we can derive that $u$ is uniformly small. Details are lengthy but straightforward, and are left to the reader.

\subsection{The lowest eigenvalue of $L$ on $\Sigma_{T}$}

Theorem 2 The lowest eigenvalue $\lambda_{0}=\lambda_{0}(T)$ for $L$ on $\Sigma_{T}$ satisfies $\lambda_{0} \geq C T^{-2}$ for some constant $C>0$ which is independent of $T$.

Proof of Theorem 2 when the restriction of $\gamma_{T}$ to $C_{T}$ is the product metric: If this result were false, it would be possible to find a sequence of values $T_{j} \rightarrow \infty$ such that for the operators $L_{T_{j}}$ associated to the metrics $\gamma_{T_{j}}$, we would have $\lambda_{0}\left(T_{j}\right) T_{j}^{2} \rightarrow 0$. We now show this leads to a contradiction.

Denote the sequence of metrics and operators by $\gamma_{j}$ and $L_{j}$, respectively. Let $X_{j}$ be any one of the eigenfunctions for $L_{j}$ corresponding to the eigenspace with eigenvalue $\lambda_{0}\left(T_{j}\right)$. By rescaling, assume that $\sup _{\Sigma_{T_{j}}}\left|X_{j}\right|=1$.

The coefficients of $L_{j}$ converge uniformly on any compact subset of $\Sigma^{*}$ to the coefficients of the operator $L_{c}$ corresponding to the metric $\gamma_{c}$. Since $\left|X_{j}\right|$ is uniformly bounded, we may extract a subsequence $X_{j^{\prime}}$ which converges uniformly on compact sets in $\Sigma^{*}$ to a limit $X$. This vector field is a solution of $L_{c} X=0$ and satisfies $|X| \leq 1$.

Our first claim is that $X$ is nontrivial. To see this, relabel the subsequence as $X_{j}$ again. Suppose that the supremum of $\left|X_{j}\right|$ is attained at some point $q_{j} \in \Sigma_{T_{j}}$. If $q_{j}$ lies in $\Sigma_{T_{j}} \backslash C_{T_{j}}$ for infinitely many $j$, then again possibly passing to a subsequence $q_{j} \rightarrow q \in \Sigma^{*}$. Since $\left|X_{j}\left(q_{j}\right)\right| \equiv 1$, we get $|X(q)|=1$, and so $X \not \equiv 0$. On the other hand, suppose that $q_{j} \in C_{T_{j}}$ for all but finitely many $j$. Note that by Schauder theory, $\left|X_{j}\right|$ remains bounded away from zero on a strip $\left[a_{j}-\eta, a_{j}+\eta\right] \times S^{2} \subset C_{T_{j}}$ of width not depending on $j$, about $q_{j}$. Furthermore, $\lambda_{0}\left(T_{j}\right)<\frac{1}{8} T_{j}^{2}$ for $j$ large enough, and hence Proposition 3 (and elliptic theory) implies that $\left|X_{j}\left(q_{j}^{\prime}\right)\right| \geq \alpha>0$ for some point $q_{j}^{\prime} \in \partial C_{T_{j}}$ and some $\alpha$ not depending on $j$. Therefore in this case too we may conclude that the limit $X \not \equiv 0$ on $\Sigma^{*}$. 
By the analysis of $\S 3.1$, if $X$ is any solution of $L_{c} X=0$ on $\Sigma^{*}$ (with respect to the metric $\gamma_{c}$ ) which is bounded along the cylindrical end, then it can be written as a sum $X=X_{\infty}+Z$, where $X_{\infty}$ extends to a bounded solution of $L X_{\infty}=0$ on the entire cylinder $\mathbb{R} \times S^{2}$ (with product metric) and $Z$ decays exponentially as $t \rightarrow \infty$.

Our second claim is that, assuming the nondegeneracy of $\Sigma$ so that there are no conformal Killing fields vanishing at both points $p_{j}$, then any bounded vector field $X$ on $\Sigma^{*}$ which satisfies $L_{c} X=0$ necessarily vanishes identically. To see this, observe from our earlier result about elements in the nullspace of $L_{c}$ which are bounded on the cylinder, all such solutions are also annihilated by $\mathcal{D}$. (This is not true if we also admit the linearly growing solutions.) Thus the term $X_{\infty}$ in the decomposition of $X$ above is annihilated by $\mathcal{D}$, and hence $\mathcal{D} X=\mathcal{D} Z$ decays exponentially along the cylindrical ends. Therefore, no boundary contribution appears in the integration by parts

$$
0=\left\langle L_{c} X, X\right\rangle=\left\langle\mathcal{D}^{*} \mathcal{D} X, X\right\rangle=\|\mathcal{D} X\|^{2},
$$

so that $\mathcal{D} X \equiv 0$. Thus $X$ is a conformal Killing field on $\Sigma^{*}$ which is bounded with respect to the metric $\gamma_{c}$; changing back to the old polar variables $r_{j}=e^{-t_{j}}$ and $\theta$ on each ball $B_{j}$ shows that $X$ is bounded with respect to the original metric $\gamma$ on $\Sigma$, and in fact vanishes at both points $p_{j}$. It is therefore in the (distributional) nullspace of $L$ on $\Sigma$, and hence by elliptic regularity extends smoothly to a solution on all of $\Sigma$. It would thus be a nontrivial conformal Killing field on the manifold $\Sigma$, vanishing at both $p_{1}$ and $p_{2}$, and we have assumed that such a field does not exist. This completes the proof.

Proof of Theorem 2 in general: We may follow the outline of the preceding proof at almost every point. Thus we suppose the result false and choose a sequence of eigenfunctions $X_{j}$ with corresponding very small eigenvalues $\mu_{j}$, for some sequence $T_{j} \rightarrow \infty$. Supposing that $\left|X_{j}\right| \leq 1$, we again attempt to pass to a limit and obtain a contradiction. If $\left|X_{j}\right|$ remains bounded away from zero on some fixed compact set near the ends, then we argue as before. The difficulty, however, is when $\left|X_{j}\right|$ remains bounded away from zero in some portion of the cylinder $C_{T_{j}}$ which recedes from either end. To handle this, we would like to use the same sort of argument as in the proof for the product case. Namely, we would like to assert that if $\left|X_{j}\right|$ tends to zero near the ends of $C_{T_{j}}$, then it must tend to zero uniformly along all of this cylindrical segment. We shall now prove this using Proposition 1 .

The metric $\gamma_{T}$ differs from the product metric on $C_{T}$ by a term of size $e^{-T / 2} \cosh s$, and therefore the operator $L$ computed relative to $\gamma_{T}$ differs from the corresponding operator $L_{0}$, computed relative to the product metric, by an error term $E_{T}$ with coefficients of this same size. We may rewrite the equation for $X_{j}$ as

$$
L_{0} X_{j}=\mu_{j} X_{j}+F_{j},
$$

where $F_{j}=-E_{T_{j}} X_{j}$. Now suppose that we are in the difficult situation: that $\left|X_{j}\right|$ is uniformly small on any fixed neighborhood of the ends of the cylinder. Then it is clear that $F_{j}$ satisfies precisely the estimates in the statement of Proposition 1 . The solution $X_{j}$ may be decomposed into a sum $U_{j}+V_{j}$, where $U_{j}$ satisfies the homogeneous problem $L_{0} U_{j}=\mu_{j} U_{j}$ and $U_{j}( \pm T / 2)=X_{j}( \pm T / 2)$, while $V_{j}$ satisfies the inhomogeneous problem with vanishing boundary conditions. Clearly both of these summands are uniformly small near the ends of $C_{T_{j}}$. The uniform smallness of $\left|U_{j}\right|$ on the entire cylinder now 
follows from Proposition 3, while the uniform smallness of $\left|V_{j}\right|$ follows from Proposition 4 . Therefore it is impossible that $X_{j}$ is large in the middle of $C_{T_{j}}$ but small at the ends. This completes the proof.

\subsection{Estimates on Hölder spaces}

We have shown that the lowest eigenvalue of the operator $L$ on $\Sigma_{T}$ is bounded below by $C T^{-2}$, uniformly as $T \rightarrow \infty$. Hence the norm of $G=L^{-1}$, as a bounded operator on $L^{2}\left(\Sigma_{T}\right)$, blows up no faster than $C^{\prime} T^{2}$. However, it is more convenient for us to use Hölder spaces because of the nonlinear nature of the problem, and we show now that this $L^{2}$ estimate may be converted to a slightly weaker estimate on appropriately defined Hölder spaces.

Definition 2 Consider the cover of $\Sigma_{T}$ given by the sets $\Sigma_{R / 2}^{*}$ and $C_{T}$, and for any vector field $X$ on $\Sigma_{T}$, let $X^{\prime}$ and $X^{\prime \prime}$ denote the restrictions of $X$ to these two subsets, respectively. Also, for $-T / 2+1 \leq a \leq T / 2-1$, define $N_{a}=[a-1, a+1] \times S^{2} \subset C_{T}$. Then for any $k \in \mathbb{N}$ and $\alpha \in(0,1)$, define

$$
\|X\|_{k, \alpha}=\left\|X^{\prime}\right\|_{k, \alpha}+\sup _{-T / 2+1 \leq a \leq T / 2-1}\left\|X^{\prime \prime}\right\|_{k, \alpha, N_{a}} .
$$

The local Hölder norms here are computed with respect to the metric $\gamma_{T}$.

Corollary 1 Let $W \in \mathcal{C}^{k, \alpha}\left(\Sigma_{T}\right)$ and suppose that $X$ is the unique solution to $L X=W$, as provided by Theorem $\mathrm{G}$. Then for some constant $C$ independent of $W$ and $T$,

$$
\|X\|_{k+2, \alpha} \leq C T^{3}\|W\|_{k, \alpha} .
$$

Proof: First, by Theorem 2, $\|X\|_{L^{2}} \leq C T^{2}\|W\|_{L^{2}}$. Next, since the volume of $\Sigma_{T}$ is bounded by $C T$, we have $\|W\|_{L^{2}} \leq T\|W\|_{0, \alpha}$. In addition, local Schauder estimates for $L_{c}$ give $\|X\|_{2, \alpha} \leq C\left(\|W\|_{0, \alpha}+\|X\|_{L^{2}}\right)$. Putting these together yields

$$
\|X\|_{2, \alpha} \leq C T^{3}\|W\|_{0, \alpha}
$$

which is the desired estimate when $k=0$. The estimate when $k>0$ requires another application of the local Schauder estimates.

\subsection{Correcting $\mu_{T}$ to be transverse-traceless}

Recall now that $\mu_{T}$ is constructed by patching together $\mu_{c}$ using cutoff functions in the center $Q$ of $C_{T}$, and the metric $\gamma_{T}$ is constructed from $\gamma_{c}$ the same way. The definition (11) implies that on $Q$

$$
\left|\mu_{T}\right|_{\gamma_{T}} \leq C \psi_{T}^{6}|\mu|_{\gamma}
$$

with equality (for $C=1$ ) on $\Sigma_{T} \backslash Q$. Since $\mu_{c}$ is divergence-free with respect to $\gamma_{c}$, the 'error term' $\operatorname{div} \gamma_{T} \mu_{T}$ arises from two sources: the cutoff functions used to define $\mu_{T}$ and 
those used to define $\gamma_{T}$. This error term is clearly supported in the center $Q$ of $C_{T}$, and it is not hard to see that

$$
\left\|\operatorname{div}_{\gamma_{T}} \mu_{T}\right\|_{k-1, \alpha} \leq C^{\prime}\left\|\mu_{T}\right\|_{k, \alpha} \leq C^{\prime \prime}\left\|\psi_{T}^{6}\right\|_{k, \alpha}
$$

the last inequality following from (14) since $|\mu|_{\gamma} \leq C$. Recalling that $\psi_{T} \sim e^{-t_{j} / 2}$ and each $t_{j} \sim T / 2$ on $Q$, and letting $W$ be the vector field dual to $\operatorname{div}_{\gamma_{T}} \mu_{T}$, then we conclude that

$$
\|W\|_{k+1, \alpha} \leq C e^{-3 T / 2} .
$$

Proposition 5 There exists a tensor $\sigma_{T}$ on $\Sigma_{T}$ with

$$
\left\|\sigma_{T}\right\|_{k, \alpha} \leq C T^{3} e^{-3 T / 2}
$$

and such that $\tilde{\mu}_{T}=\mu_{T}-\sigma_{T}$ is transverse-traceless with respect to $\gamma_{T}$.

Proof: As indicated in the beginning of this section, we first solve the equation $L X=W$ on $\Sigma_{T}$, with $W=\operatorname{div} \gamma_{T} \mu_{T}$, and then set $\sigma_{T}=\mathcal{D} X$. The operators $\mathcal{D}$ and $L$ here are both computed with respect to $\gamma_{T}$. The estimates for $\sigma_{T}$ and $\tilde{\mu}_{T}$ follow from (15).

\section{Estimates on the approximate solution}

Our overall goal in this paper is to modify the approximate initial data set $\left(\gamma_{T}, \mu_{T}, \psi_{T}\right)$, defined on the manifold $\Sigma_{T}$, to a genuine initial data set $\left(\gamma_{T}, \tilde{\mu}_{T}, \tilde{\psi}_{T}\right)$, so that $\tilde{\mu}_{T}$ is transverse-traceless with respect to $\gamma_{T}$ and $\tilde{\psi}_{T}$ satisfies the Lichnerowicz equation relative to $\gamma_{T}$ and $\tilde{\mu}_{T}$. Thus far we have accomplished the first of these tasks, and so what remains is to show how to modify the conformal factor $\psi_{T}$ to $\tilde{\psi}_{T}=\psi_{T}+\eta_{T}$ so that $\tilde{\psi}_{T}$ satisfies the Lichnerowicz equation. There are three separate steps involved in this. In the first we must calculate the 'error term' $E_{T}$, which measures the deviation of $\psi_{T}$ from solving the Lichnerowicz equation. After that we must analyze the mapping properties of the linearization of this equation, calculated at $\psi_{T}$, uniformly as $T \rightarrow \infty$. Finally, these are put together to carry out the contraction mapping argument to produce the exact solution $\tilde{\psi}_{T}$. These steps are taken up in this and the next two sections, successively.

To begin then, denote by $\mathcal{N}_{T}$ the Lichnerowicz operator with respect to $\left(\gamma_{T}, \tilde{\mu}_{T}\right)$ :

$$
\mathcal{N}_{T}(\psi)=\Delta_{T} \psi-\frac{1}{8} R_{T} \psi+\frac{1}{8}\left|\tilde{\mu}_{T}\right|^{2} \psi^{-7}-\frac{1}{12} \tau^{2} \psi^{5} .
$$

The Laplacian and scalar curvature and norm squared of $\tilde{\mu}_{T}$ are all computed with respect to $\gamma_{T}$ here. We define the error term

$$
E_{T}=\mathcal{N}_{T}\left(\psi_{T}\right)
$$

which measures the deviation of $\psi_{T}$ from being an exact solution.

Proposition 6 For every $k$ and $\alpha$ there exists a constant $C$ which does not depend on T such that $\left\|E_{T}\right\|_{k, \alpha} \leq C e^{-T / 2}$. 
The proof of this proposition involves a careful accounting of the various terms in $\mathcal{N}_{T}\left(\psi_{T}\right)$.

We begin by noting that away from the neck region $C_{T}$, the estimate is nearly trivial. In fact, on $\Sigma_{R}^{*}, \gamma_{T}=\gamma_{c}, \psi_{T}=\psi_{c}$ and $\tilde{\mu}_{T}=\mu_{c}+\mathcal{O}\left(T^{3} e^{-3 T / 2}\right)$. Inserting these into the Lichnerowicz equation, and using that $\left(\gamma_{c}, \mu_{c}, \psi_{c}\right)$ is an exact solution, we have that

$$
\left|E_{T}\right| \leq C T^{3} e^{-3 T / 2} \leq C e^{-T / 2}
$$

along with all its derivatives, as claimed.

We divide the tube $C_{T}$ up into three regions: the center $Q=[-1,1] \times S^{2}$, and the regions to the left and right of this, $[-T / 2,-1] \times S^{2} \equiv C_{T}^{(1)}$ and $[1, T / 2] \times S^{2} \equiv C_{T}^{(2)}$. We also recall the notation used in the construction of the approximate solution that $\gamma_{j}$, $\mu_{j}$ and $\psi_{j}$ denote the restrictions of $\gamma_{c}, \mu_{c}$ and $\psi_{c}$ to the balls $B_{j}$, and hence, by a small abuse of notation, to the tube $C_{T}$ as well. Thus $\left(\gamma_{1}, \mu_{1}, \psi_{1}\right)$ represents data 'coming in from the left', while $\left(\gamma_{2}, \mu_{2}, \psi_{2}\right)$ represents data 'coming in from the right'. Recall also that $\gamma_{T}$ and $\mu_{T}$ agree with $\gamma_{j}$ and $\mu_{j}$ on $C_{T}^{(j)}$, whereas $\psi_{T}$ is a sum of two terms, one much larger than the other, in each of these side regions.

We begin by listing some trivial estimates which hold in all of $C_{T}$, and which follow from the construction of the approximate solution and from (16):

a) $\gamma_{T}=d s^{2}+h+\mathcal{O}\left(e^{-T / 2} \cosh s\right)$,

b) $\psi_{T} \sim 2 e^{-T / 4} \cosh (s / 2)$,

c) $\left|\mu_{T}\right| \sim \psi_{T}^{6} \leq C e^{-3 T / 2}(\cosh (s / 2))^{6}$,

d) $\left|\tilde{\mu}_{T}-\mu_{T}\right| \leq C T^{3} e^{-3 T / 2}$.

We shall also repeatedly use the fact that

$$
\Delta_{\gamma_{j}} \psi_{j}-\frac{1}{8} R\left(\gamma_{j}\right) \psi_{j}+\frac{1}{8}\left|\mu_{j}\right|^{2} \psi_{j}^{-7}-\frac{1}{12} \tau^{2} \psi_{j}^{5} \equiv 0 .
$$

We begin by estimating $E_{T}$ in $Q$. The main observation concerning this estimate is that we have a decomposition

$$
\psi_{T}=\psi_{1}+\psi_{2}
$$

valid in $Q$. In addition, it follows from property (a) above and from the definition of $\gamma_{T}$ that

$$
\Delta_{T}-\frac{1}{8} R_{T}=\Delta_{\gamma_{j}}-\frac{1}{8} R_{\gamma_{j}}+\mathcal{O}\left(e^{-T / 2}\right)
$$

for $j=1$ or 2 . On $Q$, we may therefore expand $\left(\Delta_{T}-\frac{1}{8} R_{T}\right) \psi_{T}$ as a sum of two terms. Since on $Q, \psi_{j}=\mathcal{O}\left(e^{-T / 4}\right)$, we see from (19) that we have

$$
\left(\Delta_{T}-\frac{1}{8} R_{T}\right) \psi_{T}=\sum_{j=1}^{2}\left(-\frac{1}{8}\left|\mu_{j}\right|^{2} \psi_{j}^{-7}+\frac{1}{12} \tau^{2} \psi_{j}^{5}\right)+\mathcal{O}\left(e^{-3 T / 4}\right) .
$$

From this, using the estimate that $\left|\mu_{j}\right|^{2}=\mathcal{O}\left(e^{-3 T}\right)$ in $Q$, we easily conclude that

$$
\left(\Delta_{T}-\frac{1}{8} R_{T}\right) \psi_{T}=\mathcal{O}\left(e^{-3 T / 4}\right)
$$


As for the other terms in the Lichnerowicz equation, using properties (b), (c) and (d) above we easily get suitable estimates for them in this region as well. In particular, we have $\left|\tilde{\mu}_{T}\right|^{2} \psi_{T}^{-7}=\mathcal{O}\left(T^{6} e^{-5 T / 4}\right)$ and $\tau^{2} \psi_{T}^{5}=\mathcal{O}\left(e^{-5 T / 4}\right)$. Hence in $Q$, we obtain $\left|E_{T}\right|=$ $\mathcal{O}\left(e^{-3 T / 4}\right)$, which is even better than the estimate we have stated.

The estimates in $C_{T}^{(1)}$ and $C_{T}^{(2)}$ are nearly identical to one another, so it suffices to concentrate on just one of these, let us say in $C_{T}^{(2)}$ to be concrete. In this region, $\psi_{T}=\psi_{2}+\chi \psi_{1}$, where $\chi$ is a function cutting $\psi_{1}$ off to zero at the far end of $C_{T}$, where $s \approx T / 2$. In this region

$$
\psi_{1}=e^{-T / 4} e^{-s / 2}+\mathcal{O}\left(e^{-T / 4} e^{-3 s / 2}\right), \quad \psi_{2} \sim e^{-T / 4} e^{s / 2},
$$

and in addition

$$
\Delta_{T}-\frac{1}{8} R_{T}=\Delta_{0}-\frac{1}{8} R_{0}+\mathcal{O}\left(e^{-T / 2} \cosh s\right)
$$

Therefore

$$
\left(\Delta_{T}-\frac{1}{8} R_{T}\right)\left(\chi \psi_{1}\right)=e^{-3 T / 4} \cosh s e^{-s / 2}+\mathcal{O}\left(e^{-T / 2}\right)=\mathcal{O}\left(e^{-T / 2}\right) .
$$

Next, $\psi_{T}=\psi_{2}\left(1+\chi \psi_{1} / \psi_{2}\right)$ and hence

$$
\psi_{T}^{-7}=\psi_{2}^{-7}+\mathcal{O}\left(e^{7 T / 4} e^{-9 s / 2}\right)
$$

and

$$
\psi_{T}^{5}=\psi_{2}^{5}+\mathcal{O}\left(e^{-5 T / 4} e^{3 s / 2}\right)
$$

Furthermore, we have here

$$
\left|\tilde{\mu}_{T}\right|^{2}=\left|\mu_{2}\right|^{2}+\mathcal{O}\left(T^{3} e^{-3 T / 2}\right)
$$

Putting these estimates all together yields $\left|E_{T}\right| \leq C e^{-T / 2}$ on $C_{T}^{(2)}$. With a similar estimate on $C_{T}^{(1)}$ we conclude that $\left|E_{T}\right| \leq C e^{-T / 2}$ on all of $C_{T}$. The estimates on the derivatives of $E_{T}$ follow similarly.

\section{The linearization of the Lichnerowicz operator}

Our main goal now is to find a correction term $\eta_{T}$ for the approximate solution $\psi_{T}$ so that the sum $\psi_{T}+\eta_{T}$ solves the Lichnerowicz equation $\mathcal{N}_{T}\left(\psi_{T}+\eta_{T}\right)=0$ on $\Sigma_{T}$. This will be done in the next section using a contraction mapping argument. In preparation for this we establish in this section good estimates for the mapping properties of the linearization $\mathcal{L}_{T}$ of this operator $\mathcal{N}_{T}$ about the approximate solution $\psi_{T}$; as usual, keeping track of behavior as $T \rightarrow \infty$.

We first record that the linearization $\mathcal{L}$ of the Lichnerowicz operator on $\Sigma$ with respect to the initial data set $(\gamma, \mu)$ is given by

$$
\mathcal{L}=\Delta_{\gamma}-\frac{1}{8}\left(R(\gamma)+7|\mu|_{\gamma}^{2}+\frac{10}{3} \tau^{2}\right),
$$


but using the fact that the pair $(\gamma, \mu)$ satisfies the constraint equations, we can rewrite this as

$$
\mathcal{L}=\Delta_{\gamma}-\left(|\mu|_{\gamma}^{2}+\frac{1}{3} \tau^{2}\right)
$$

By standard elliptic theory (for $\Sigma$ compact),

$$
\mathcal{L}: \mathcal{C}^{k+2, \alpha}(\Sigma) \rightarrow \mathcal{C}^{k, \alpha}(\Sigma)
$$

is a Fredholm mapping of index zero for every $k \in \mathbb{N}$ and $\alpha \in(0,1)$. Furthermore, since we are assuming that $\Pi \not \equiv 0$, which is equivalent to $\tau \neq 0$ or $\mu \not \equiv 0$, the term of order zero here is nonpositive and not identically zero and so the maximum principle implies that there are no nontrivial solutions of $\mathcal{L} \phi=0$. Consequently the cokernel is also trivial, and we see that (20) is always an isomorphism.

Our first goal in this section is to establish that the same property is true for the linearization $\mathcal{L}_{T}$ on $\Sigma_{T}$, which is given by

$$
\mathcal{L}_{T}=\Delta_{\gamma_{T}}-\frac{1}{8}\left(R\left(\gamma_{T}\right)+7\left|\tilde{\mu}_{T}\right|^{2} \psi_{T}^{-8}+\frac{10}{3} \tau^{2} \psi_{T}^{4}\right) .
$$

This operator is elliptic and Fredholm of index zero acting on the Hölder spaces introduced in $§ 3.3$; unfortunately, the term of order zero here is no longer necessarily nonpositive since it can no longer be rewritten using the constraint equations, and we do not have control on the sign of $R\left(\gamma_{T}\right)$ near the boundary of $\Sigma_{R}^{*}$. Therefore, the triviality of the nullspace of $\mathcal{L}_{T}$ when $T$ is large enough requires further argument. We must also show that the norm of the inverse operator is uniformly bounded as $T \rightarrow \infty$. This last issue is complicated by the following considerations. We shall be using this solution operator in an iterative scheme to produce the correction term $\eta_{T}$ for the approximate solution $\psi_{T}$. Because of the term $\psi^{-7}$ in the Lichnerowicz equation, it is quite important that $\eta_{T}$ be substantially smaller than $\psi_{T}$. Furthermore, we also desire that $\eta_{T}$ vanish exponentially as $T \rightarrow \infty$ on the 'body' $\Sigma_{R}^{*}$, away from the scene of the surgery. To ensure that both of these requirements are met in the iteration scheme, we establish precise estimates for the solution operator for $\mathcal{L}_{T}$. This necessitates using slight alterations of the Hölder spaces which incorporate a weight factor along the neck. Thus we first define these weighted Hölder spaces and then take up the matters of the existence and uniformity of the inverse of $\mathcal{L}_{T}$ on these spaces.

Definition 3 Let $w_{T}$ be an everywhere positive smooth function on $\Sigma_{T}$ which equals $e^{-T / 4} \cosh (s / 2)$ on $C_{T}$ and which is uniformly bounded away from zero on $\Sigma_{R}^{*}$. (We may as well also assume that $w_{T} \equiv 1$ on $\left.\Sigma_{2 R}^{*}.\right)$ Now, for any $\delta \in \mathbb{R}$, and any $\phi \in \mathcal{C}^{k, \alpha}\left(\Sigma_{T}\right)$, set

$$
\|\phi\|_{k, \alpha, \delta}=\left\|w_{T}^{-\delta} \phi\right\|_{k, \alpha},
$$

and let $\mathcal{C}_{\delta}^{k, \alpha}\left(\Sigma_{T}\right)$ denote the corresponding normed space.

Proposition 7 Fix any $\delta \in \mathbb{R}$. For $T$ sufficiently large, the mapping

$$
\mathcal{L}_{T}: \mathcal{C}_{\delta}^{k+2, \alpha}\left(\Sigma_{T}\right) \longrightarrow \mathcal{C}_{\delta}^{k, \alpha}\left(\Sigma_{T}\right)
$$

is an isomorphism. 
Proof: The action of $\mathcal{L}_{T}$ on $\mathcal{C}_{\delta}^{k+2, \alpha}\left(\Sigma_{T}\right)$ is equivalent to the action of the conjugated operator $w_{T}^{-\delta} \mathcal{L}_{T} w_{T}^{\delta}$ on the unweighted space $\mathcal{C}^{k+2, \alpha}$. As $\delta$ varies, these mappings are all Fredholm of index zero. Furthermore, any element of the nullspace of $\mathcal{L}_{T}$ is in any one of the spaces $\mathcal{C}_{\delta}^{k+2, \alpha}$. Therefore, to prove that any one of these maps is an isomorphism, it suffices to show that the nullspace of $\mathcal{L}_{T}$ is trivial when $\delta=0$. In addition, by elliptic regularity, it even suffices to show that there is no element $\eta \in \mathcal{C}^{0}\left(\Sigma_{T}\right)$ such that $\mathcal{L}_{T} \eta=0$.

Assume this is not the case, so that there exists a sequence $T_{j} \rightarrow \infty$ and functions $\eta_{j} \in$ $C^{0}\left(\Sigma_{T_{j}}\right)$ satisfying $\mathcal{L}_{T_{j}}\left(\eta_{j}\right)=0$. Write $\mathcal{L}_{T_{j}}=\mathcal{L}_{j}$ and normalize $\eta_{j}$ so that $\sup \left|\eta_{j}\right|=1$. As in the proof of Theorem 2, we shall show first that we may extract a nontrivial limiting function $\eta$, and then show that the existence of this function leads to a contradiction.

Suppose first that the supremum of $\left|\eta_{j}\right|$ on $\Sigma_{R}^{*}$ remains bounded away from zero, at least for infinitely many $j$. Then using local elliptic theory to control higher order derivatives, we may extract a subsequence $\eta_{j^{\prime}}$ which converges to some limiting function $\eta \not \equiv 0$ on $\Sigma^{*}$. Since the coefficients of $\mathcal{L}_{j}$ converge on compact sets to those of $\mathcal{L}_{c}$, the linearization of the Lichnerowicz operator with respect to the initial data set $\left(\gamma_{c}, \mu_{c}\right)$ at $\psi=\psi_{c}$, we see that

$$
\mathcal{L}_{c} \eta=\Delta_{\gamma_{c}} \eta-\frac{1}{8}\left(R\left(\gamma_{c}\right)+7\left|\mu_{c}\right|^{2} \psi_{c}^{-8}+\frac{10}{3} \tau^{2} \psi_{c}^{4}\right) \eta=0
$$

The first two terms, $\Delta_{\gamma_{c}}-\frac{1}{8} R\left(\gamma_{c}\right)$, constitute the conformal Laplacian for $\gamma_{c}$. Since $\gamma_{c}=\psi_{c}^{-4} \gamma$, the familiar conformal covariance property of this operator leads to

$$
\Delta_{\gamma_{c}} \eta-\frac{1}{8} R\left(\gamma_{c}\right) \eta=\psi_{c}^{5}\left(\Delta_{\gamma}\left(\psi_{c}^{-1} \eta\right)-\frac{1}{8} R(\gamma) \psi_{c}^{-1} \eta\right)
$$

Using this in (22), as well as the fact that $\left|\mu_{c}\right|_{\gamma_{c}}^{2}=\psi_{c}^{12}|\mu|_{\gamma}^{2}$, and dividing through by $\psi_{c}^{5}$, we conclude that $\mathcal{L} u=0$ on $\Sigma^{*}$, where $u=\eta / \psi_{c}$. (Note that $\mathcal{L}$ in this equation is the operator which extends smoothly across the $p_{j}$.) Next, $\eta$ is bounded on $\Sigma^{*}$ and $\psi_{c} \sim r_{j}^{1 / 2}$ near $p_{j}$, and so, in $B_{j},|u| \leq C r_{j}^{-1 / 2}$. This singularity is weaker than that of the Green's function in three dimensions, and so $u$ extends to a weak solution of $\mathcal{L} u=0$ on all of $\Sigma$. But we have already remarked earlier that the operator $\mathcal{L}$ on $\Sigma$ has trivial nullspace, which means that $u \equiv 0$. This is a contradiction.

It remains to address the case in which the supremum of $\left|\eta_{j}\right|$ on $\Sigma_{R}^{*}$ converges to zero. In fact, if $\sup \left|\eta_{j}\right|$ is attained at some point $q_{j} \in \Sigma_{T_{j}}$, then we may apply the same argument as before if $q_{j}$ remains within a bounded distance of the end of the cylindrical piece $C_{T_{j}}$. If this distance tends to infinity, then choose a new linear coordinate $s^{\prime}$ which is centered at the point $q_{j}$ (so that $s^{\prime}=0$ at that point) and is a translation of the coordinate $s$. Using local elliptic theory again we may extract a subsequence which converges on any compact set, along with all its derivatives, to some nontrivial function $\eta$ defined on the complete cylinder $\mathbb{R} \times S^{2}$. Furthermore, the metric $\gamma_{T_{j}}$ converges in this limit to the product metric $\gamma_{0}=\left(d s^{\prime}\right)^{2}+h, R\left(\gamma_{T_{j}}\right) \rightarrow 2$, and the terms $\left|\mu_{T_{j}}\right|^{2} \psi_{T_{j}}^{-8}$ and $\psi_{T_{j}}^{4}$ both converge to zero. Hence $\mathcal{L}_{j}$ converges locally on compact sets to $\Delta_{\gamma_{0}}-1 / 4$. Thus we have obtained in this limit a nontrivial bounded solution of the equation

$$
\mathcal{L}_{\gamma_{0}} \eta=\Delta_{\gamma_{0}} \eta-\frac{1}{4} \eta=0
$$


on the entire cylinder $\mathbb{R} \times S^{2}$, and such a solution obviously cannot exist. Hence this case too leads to a contradiction.

This completes the proof of Proposition 0 .

For $T$ sufficiently large, let $\mathcal{G}_{T}$ denote the inverse of $\mathcal{L}_{T}$ acting on $\mathcal{C}_{\delta}^{k, \alpha}$. In other words, we have

$$
\mathcal{G}_{T}: \mathcal{C}_{\delta}^{k, \alpha}\left(\Sigma_{T}\right) \longrightarrow \mathcal{C}_{\delta}^{k+2, \alpha}\left(\Sigma_{T}\right)
$$

with $\mathcal{L}_{T} \mathcal{G}_{T}=\mathcal{G}_{T} \mathcal{L}_{T}=I$. Although of course $\mathcal{G}_{T}$ depends on the chosen weight $\delta$, we suppress this dependence in the notation.

Proposition 8 If $0<\delta<1$, then the norm of the operator $\mathcal{G}_{T}$ in (25) is uniformly bounded as $T \rightarrow \infty$.

Proof: The strategy is the same as before. If the result were false, then there would exist a sequence $T_{j} \rightarrow \infty$ and functions $f_{j} \in \mathcal{C}_{\delta}^{k, \alpha}\left(\Sigma_{T_{j}}\right)$ such that, writing $\mathcal{G}_{j}=\mathcal{G}_{T_{j}}$,

$$
\left\|f_{j}\right\|_{k, \alpha, \delta} \rightarrow 0 \quad \text { and } \quad\left\|\mathcal{G}_{j}\left(f_{j}\right)\right\|_{k+2, \alpha, \delta}=1
$$

as $j \rightarrow \infty$.

Setting $v_{j}=\mathcal{G}_{j}\left(f_{j}\right)$, we argue exactly as in the preceding proof. Either some subsequence of the $v_{j}$ converges to a bounded, nontrivial function $v$ on $\Sigma^{*}$ which satisfies $\mathcal{L}_{c} v=0$, which leads to a contradiction, or else some subsequence converges on the cylinder. This second case is precisely why we have introduced the weight function $w_{T_{j}}^{\delta}$.

Write $w_{T_{j}}=w_{j}$ and $C_{T_{j}}=C_{j}$. Since $\left\|v_{j}\right\|_{k+2, \alpha, \delta} \equiv 1$, we have

$$
1 \geq \sup _{j} \sup _{C_{j}} w_{j}^{-\delta}\left|v_{j}\right| \geq c>0
$$

Suppose that $\sup _{C_{j}} w_{j}^{-\delta}\left|v_{j}\right|$ is attained at some point $q_{j} \in C_{j}$. We may also assume that $\left|v_{j}\right| \rightarrow 0$ on any fixed compact set of $\Sigma^{*}$ away from $C_{j}$. Now, by assumption $\left|v_{j}\right| \leq w_{j}^{\delta}$, and if we renormalize by setting $\tilde{v}_{j}=e^{T_{j} / 4} v_{j}$, then this becomes

$$
\left|\tilde{v}_{j}(s, \theta)\right| \leq(\cosh (s / 2))^{\delta}
$$

on $C_{T_{j}}$, with equality attained at the point $q_{j}$. Writing $q_{j}=\left(s_{j}, \theta_{j}\right)$, then we renormalize yet again, setting $\hat{v}_{j}\left(s^{\prime}, \theta\right)=\tilde{v}_{j}\left(s^{\prime}+s_{j}, \theta\right) /\left(\cosh \left(s_{j} / 2\right)\right)^{\delta}$. Hence

$$
\left|\hat{v}_{j}\left(s^{\prime}, \theta\right)\right| \leq\left(\frac{\cosh \frac{1}{2}\left(s^{\prime}+s_{j}\right)}{\cosh \frac{1}{2} s_{j}}\right)^{\delta},
$$

with equality at the point $\left(s^{\prime}, \theta\right)=\left(0, \theta_{j}\right)$. In particular, $\hat{v}_{j}\left(0, \theta_{j}\right)=1$. Now we can pass to a limit. If $s_{j} \rightarrow \pm \infty$, then the right hand side here converges to $e^{\delta s^{\prime} / 2}$ or $e^{-\delta s^{\prime} / 2}$, while if $s_{j}$ remains bounded, then the right side converges to a function which is bounded by $C\left(\cosh \left(s^{\prime} / 2\right)\right)^{\delta}$ for some $C>0$, while the left side converges to a nontrivial function $\hat{v}$ which satisfies $\mathcal{L}_{\gamma_{0}} \hat{v}=0$. But any solution of this equation blows up at least like $e^{\left|s^{\prime}\right| / 2}$ either as $s^{\prime} \rightarrow \infty$ or as $s^{\prime} \rightarrow-\infty$. Since $0<\delta<1$, this rate of growth is incompatible with the previous inequalities, and so we reach a contradiction once again. 


\section{Proof of the main Theorem}

We wish to solve

$$
\mathcal{N}_{T}\left(\psi_{T}+\eta_{T}\right)=0
$$

Expanding $\mathcal{N}_{T}\left(\psi_{T}+\eta\right)$ in a Taylor series about $\eta=0$ we have

$$
\mathcal{N}_{T}\left(\psi_{T}+\eta\right)=E_{T}+\mathcal{L}_{T}(\eta)+\mathcal{Q}_{T}(\eta),
$$

where $E_{T}$ and $\mathcal{L}_{T}$ are given by (18) and (21) respectively, and

$$
\begin{aligned}
\mathcal{Q}_{T}(\eta)= & \mathcal{N}_{T}\left(\psi_{T}+\eta\right)-E_{T}-\mathcal{L}_{T}(\eta) \\
= & \frac{1}{8}\left|\tilde{\mu}_{T}\right|_{\gamma_{T}}^{2}\left(\left(\psi_{T}+\eta\right)^{-7}-\psi_{T}^{-7}+7 \psi_{T}^{-8} \eta\right) \\
& -\frac{1}{12} \tau^{2}\left(\left(\psi_{T}+\eta\right)^{5}-\psi_{T}^{5}-5 \psi_{T}^{4} \eta\right) \\
= & \frac{1}{8}\left|\tilde{\mu}_{T}\right|_{\gamma_{T}}^{2} \psi_{T}^{-7}\left(\left(1+\frac{\eta}{\psi_{T}}\right)^{-7}-1+7 \frac{\eta}{\psi_{T}}\right) \\
& -\frac{1}{12} \tau^{2}\left(10 \psi_{T}^{3} \eta^{2}+10 \psi_{T}^{2} \eta^{3}+5 \psi_{T} \eta^{4}+\eta^{5}\right) .
\end{aligned}
$$

is the "quadratically vanishing" nonlinearity. If we require that $\eta \in \mathcal{C}_{\delta}^{k+2, \alpha}\left(\Sigma_{T}\right)$ satisfy $|\eta|<c \psi_{T}$ for some constant $c<1$, then from the expansion above we easily obtain an estimate of the form

$$
\left\|\mathcal{Q}_{T}(\eta)\right\|_{k+2, \alpha, \delta} \leq C\|\eta\|_{k+2, \alpha, \delta}^{2}
$$

for some constant $C$ independent of $T$. Equation (26) is equivalent to

$$
\eta_{T}=-\mathcal{G}_{T}\left(E_{T}+\mathcal{Q}_{T}\left(\eta_{T}\right)\right)
$$

where $\mathcal{G}_{T}$ is the inverse of $\mathcal{L}_{T}$ acting on $\mathcal{C}_{\delta}^{k+2, \alpha}\left(\Sigma_{T}\right)$. We will find a solution to this equation in a small ball about the origin in $\mathcal{C}_{\delta}^{k+2, \alpha}\left(\Sigma_{T}\right)$ for $\delta \in(0,1)$. To determine the appropriate size of the ball in which to work, note that from Proposition 6, for any $\delta \in(0,1)$, we have

$$
\left\|E_{T}\right\|_{k, \alpha, \delta} \leq C e^{-T / 4}
$$

for a constant $C$ which is independent of $T$. This suggests the following

Definition 4 Fix $k \in \mathbb{N}, \alpha \in(0,1)$ and $\delta \in(0,1)$, and let $\nu \in \mathbb{R}^{+}$. Then we define

$$
\mathbb{B}_{\nu}=\left\{u \in \mathcal{C}_{\delta}^{k+2, \alpha}\left(\Sigma_{T}\right):\|u\|_{k+2, \alpha, \delta} \leq \nu e^{-T / 4}\right\} .
$$

Thus, for any $\eta \in \mathbb{B}_{\nu}$, we have

$$
|\eta| \leq \nu e^{-T / 4} \quad \text { on } \quad \Sigma_{T} \backslash C_{T} \quad \text { and } \quad|\eta| \leq \nu e^{-\delta T / 4} \psi_{T} \quad \text { on } \quad C_{T} .
$$

In particular, when $T$ is sufficiently large, the quadratic estimate (27) is valid in $\mathbb{B}_{\nu}$. Using Proposition 8 together with (28) we have

$$
\left\|\mathcal{G}_{T}\left(E_{T}\right)\right\|_{k+2, \alpha, \delta} \leq C M e^{-T / 4}
$$

where $M$ is the bound on $\mathcal{G}_{T}$ acting on $\mathcal{C}_{\delta}^{k, \alpha}\left(\Sigma_{T}\right)$. In light of this we choose $\nu=2 C M$ so that we have $\mathcal{G}_{T}\left(E_{T}\right) \subset \mathbb{B}_{\nu / 2}$. The following Lemma follows easily from the fact that we have the quadratic estimate above on $\mathcal{Q}_{T}(\eta)$ for $\eta \in \mathbb{B}_{\nu}$. 
Lemma 1 For $\nu=2 C M$ as above and $T$ sufficiently large we have for any $\eta_{1}, \eta_{2} \in \mathbb{B}_{\nu}$

$$
\left\|\mathcal{Q}_{T}\left(\eta_{1}\right)-\mathcal{Q}_{T}\left(\eta_{2}\right)\right\|_{k+2, \alpha, \delta} \leq \frac{1}{2 M}\left\|\eta_{1}-\eta_{2}\right\|_{k+2, \alpha, \delta}
$$

We can now establish the following

Proposition 9 For $\nu$ as above and $T$ sufficiently large the map

$$
\eta \longmapsto \mathcal{T}(\eta) \equiv-\mathcal{G}_{T}\left(E_{T}+\mathcal{Q}_{T}(\eta)\right)
$$

is a contraction mapping on $\mathbb{B}_{\nu} \subset \mathcal{C}_{\delta}^{k+2, \alpha}\left(\Sigma_{T}\right)$.

Proof: Let $\eta \in \mathbb{B}_{\nu}$ be given. Taking $\eta_{1}=\eta$ and $\eta_{2}=0$ in $(30)$ we see that $-\mathcal{G}_{T}\left(\mathcal{Q}_{T}(\eta)\right) \in$ $\mathbb{B}_{\nu / 2}$. This, together with our choice of $\nu$ shows that $\mathcal{T}: \mathbb{B}_{\nu} \rightarrow \mathbb{B}_{\nu}$ as required. Moreover for $\eta_{1}, \eta_{2} \in \mathbb{B}_{\nu}$ we have

$$
\begin{aligned}
\left\|\mathcal{T}\left(\eta_{1}\right)-\mathcal{T}\left(\eta_{2}\right)\right\|_{k+2, \alpha, \delta} & =\left\|\mathcal{G}_{T}\left(E_{T}+\mathcal{Q}_{T}\left(\eta_{1}\right)\right)-\mathcal{G}_{T}\left(E_{T}+\mathcal{Q}_{T}\left(\eta_{2}\right)\right)\right\|_{k+2, \alpha, \delta} \\
& \leq M\left\|\mathcal{Q}_{T}\left(\eta_{1}\right)-\mathcal{Q}_{T}\left(\eta_{2}\right)\right\|_{k+2, \alpha, \delta} \\
& \leq \frac{1}{2}\left\|\eta_{1}-\eta_{2}\right\|_{k+2, \alpha, \delta}
\end{aligned}
$$

This completes the proof of Proposition 9 .

Theorem 1 from $\S 1.2$ is now a direct consequence of the following.

Theorem 3 The Lichnerowicz equation (20) has a unique solution $\tilde{\psi}_{T}=\psi_{T}+\eta_{T}$ with $\eta_{T} \in \mathbb{B}_{\nu} \subset \mathcal{C}_{\delta}^{k, \alpha}\left(\Sigma_{T}\right)$.

Proof: This follows immediately from the contraction mapping theorem, together with Proposition 9 .

\section{Modifications when $\Sigma$ is complete but noncompact}

Thus far we have assumed that the three-manifold $\Sigma$ is closed. However, it is also interesting from a physical perspectical to consider gluing solutions of the constraint equations on various classes of complete manifolds. Of particular interest are the two cases when $(\Sigma, \gamma)$ is either asymptotically Euclidean or asymptotically hyperbolic. For each of these cases, there are well-established results concerning the existence of CMC solutions of the Einstein constraint equation [7], [14], [2], [3]. In this section we show that with only minor modifications, the gluing constructions developed here may be extended to these other settings. Since most of the modifications are common to both cases, we begin with a more general discussion which describes the alterations and new ingredients needed to extend the arguments, modulo a few specific analytic facts. We follow this with precise statements of definitions and facts needed for these two particular cases, along with appropriate references. 


\subsection{General preamble}

Looking back, there are only a few places in the constructions and arguments of the preceding sections where truly global issues arise; elsewhere the argument localizes in the neck region. Specifically, the global structure of $\Sigma$ enters the analysis only when establishing the existence of the inverses $G_{T}$ and $\mathcal{G}_{T}$ for the vector Laplacian $L_{T}$ and the linearized Lichnerowicz operator $\mathcal{L}_{T}$, and also, more mildly, when determining the dependence on $T$ of the norms of these inverses.

Thus suppose that $(\Sigma, \gamma)$ is a complete, noncompact, three-manifold, and $(\gamma, \Pi)$ is an initial data set on $\Sigma$ with $\operatorname{tr} \Pi=\tau$ constant. In the noncompact setting we do not require that $\Pi \not \equiv 0$. Fix points $p_{1}, p_{2} \in \Sigma$ and balls $B_{j} \ni p_{j}$ and construct the approximate solutions $\left(\gamma_{T}, \mu_{T}, \psi_{T}\right)$ as in $\S 2$. Then proceed to the main part of the argument, where first $\mu_{T}$ is modified to be transverse-traceless with respect to $\gamma_{T}$ using the operator $L_{T}$, and then $\psi_{T}$ is modified so as to be a solution of the Lichnerowicz equation.

The function spaces we shall use in the two cases of interest are slightly different, and so we phrase things more abstractly in this preamble. Thus suppose that there are Banach spaces $E$ and $F$ of vector fields and $E^{\prime}$ and $F^{\prime}$ of functions - these will be familiar weighted Hölder spaces in practice - such that the mappings

$$
L: E \longrightarrow F, \quad \mathcal{L}: E^{\prime} \longrightarrow F^{\prime}
$$

for the vector Laplacian $L$ and the linearized Lichnerowicz operator $\mathcal{L}$ on $(\Sigma, \gamma)$ are both isomorphisms.

Remark 3 In the compact case, we assumed that there were no conformal Killing fields vanishing at both $p_{1}$ and $p_{2}$, and this was enough to give the invertibility and bounds for the inverse as $T \rightarrow \infty$ for $L_{T}$; likewise, the assumption that $\Pi \not \equiv 0$ was used to prove invertibility of $\mathcal{L}_{T}$. In both the asymptotically Euclidean and asymptotically hyperbolic settings, neither assumption is required because we shall use spaces of functions and vector fields which decay at infinity, and for these it is not difficult to obtain invertibility for $L_{T}$ and $\mathcal{L}_{T}$ directly. Thus the restriction that the maps (31) are both isomorphisms is not too limiting.

We must also assume that the vector fields $X \in E$ decay sufficiently so that the integration by parts

$$
\langle L X, X\rangle=\langle\mathcal{D} X, \mathcal{D} X\rangle
$$

is valid for all $X \in E$.

Since we shall always use function spaces which are localizable, i.e. preserved by multiplication by functions in $\mathcal{C}_{0}^{\infty}$, we may define new function spaces on $\Sigma_{T}$ by patching together vector fields or functions in any of these spaces with vector fields or functions in the appropriate Hölder spaces on $C_{T}$ (including functions measured with the weight factor $w^{\delta}$ as required in Propositions 7 and 8). We label these spaces $E\left(\Sigma_{T}\right), F\left(\Sigma_{T}\right)$, $E_{\delta}^{\prime}\left(\Sigma_{T}\right)$ and $F_{\delta}^{\prime}\left(\Sigma_{T}\right)$. It is clear that, presuming as above that $L$ and $\mathcal{L}$ are isomorphisms, then

$$
L_{T}: E\left(\Sigma_{T}\right) \longrightarrow F\left(\Sigma_{T}\right), \quad \mathcal{L}_{T}: E_{\delta}^{\prime}\left(\Sigma_{T}\right) \longrightarrow F_{\delta}^{\prime}\left(\Sigma_{T}\right)
$$

are Fredholm for any $T$. For simplicity, we shall usually drop the $\Sigma_{T}$ from this notation. 
Proposition 10 The operator $L_{T}: E \longrightarrow F$ is invertible when $T$ is sufficiently large. The norm of its inverse $G_{T}$, as a mapping $F \rightarrow E$, is bounded by $C T^{3}$ as $T \rightarrow \infty$.

Proposition 11 When $\delta \in(-1,1)$, the operator $\mathcal{L}_{T}: E_{\delta}^{\prime} \longrightarrow F_{\delta}^{\prime}$ is invertible, with inverse $\mathcal{G}_{T}$ uniformly bounded as a mapping $F_{\delta}^{\prime} \rightarrow E_{\delta}^{\prime}$ as $T \rightarrow \infty$.

We shall prove only the first of these two results; the second is proved in exactly the same way, but in fact is even easier to verify.

Proof of Proposition 10: Let us suppose the result is false, so there is a sequence of values $T_{j} \rightarrow \infty$, and vectors $X_{j}$ and $Y_{j}$ such that $L_{T_{j}} X_{j}=Y_{j}$, but with $\left\|X_{j}\right\|_{E} \equiv 1$ and $\left\|Y_{j}\right\|_{F}=o\left(T^{-3}\right)$. Also, write $E\left(\Sigma_{T_{j}}\right)$ and $F\left(\Sigma_{T_{j}}\right)$ simply as $E$ and $F$.

Following the argument of Theorem 2, let $q_{j}$ denote a point where $\left\|X_{j}\right\|_{E}$ attains its maximum. There are three cases to consider: the first is when $q_{j}$ lies in $C_{T_{j}}$ and the norm of $X_{j}$ on any compact set of $\Sigma^{*}$ tends to zero, the second is when $q_{j}$ remains in some fixed compact set of $\Sigma^{*}$, and the third is when $q_{j}$ leaves every compact set of $\Sigma$. In the first two cases, the argument proceeds exactly as in $\S 3.2$ and $\S 3.3$; either we produce in the limit an 'illegal' solution $X$ on the complete cylinder, or else we produce a nontrivial vector field $X$ on $\Sigma^{*}$ such that $L_{c} X=0$ there. Here $L_{c}$ is the vector Laplacian for the metric $\gamma_{c}$ on $\Sigma^{*}$ which agrees with $\gamma$ outside of the balls $B_{\ell}, \ell=1,2$, and has asymptotically cylindrical ends near these points. This solution $X$ is bounded along these cylindrical ends and it lies in the space $E$ on $\Sigma^{*} \backslash\left(B_{1} \cup B_{2}\right)$. In order to carry out the usual integration by parts, to conclude that $\mathcal{D}_{\gamma_{c}} X=0$, we use the analysis from $\S 3.2$ and the assumption (32) for $X \in E$. Hence $X$ is conformal Killing, first with respect to $\gamma_{c}$, and hence with respect to $\gamma$, on $\Sigma^{*}$. As in $\S 3.2$, it extends to a smooth solution across the marked points $p_{\ell}$, and therefore gives a nontrivial element $X \in E$ of the nullspace of $L$ on all of $\Sigma$, which is a contradiction. Thus it suffices to handle the third case.

Suppose then that $\left|X_{j}\right|$ decays to zero on every compact set of $\Sigma$. Let $\chi$ be a smooth cutoff function which vanishes near the points $p_{\ell}$ and equals one outside the balls $B_{\ell}$. Then $\tilde{X}_{j}=\chi X_{j}$ satisfies $L_{T_{j}} \tilde{X}_{j}=\chi Y_{j}+\left[L_{T_{j}}, \chi\right] X_{j} \equiv Z_{j}$. Both terms on the right decay to zero in the space $F$ on $\Sigma$ (not just $\Sigma^{*}$ ), whereas clearly $\left\|\tilde{X}_{j}\right\|_{E} \geq c>0$. However, these vector fields are all defined on $(\Sigma, \gamma)$, and $G Z_{j}=X_{j}$. Since $G$ is a bounded operator, this is again a contradiction.

\subsection{Asymptotically Euclidean initial data}

We now specialize the discussion above to the case in which $(\Sigma, \gamma)$ is asymptotically Euclidean and $(\gamma, \Pi)$ is an appropriate data set on this manifold.

Recall that a metric $\gamma$ is said to be asymptotically Euclidean (AE for short) if each end of $\Sigma$ is diffeomorphic to the exterior of a ball in $\mathbb{R}^{3}$, and in the induced coordinates the metric $\gamma$ decays to the Euclidean metric at some rate. More precisely, we require first that

$$
\Phi: \Sigma \backslash K \approx \bigsqcup\left(\mathbb{R}^{3} \backslash B_{R_{j}}\right),
$$

where the disjoint union on the right is finite. For simplicity we assume that $\Sigma$ has only one end. Then, we assume that in the naturally induced Euclidean coordinates $z$,

$$
\left|\gamma_{i j}-\delta_{i j}\right| \leq C|z|^{-\nu}
$$


along with appropriate decay of the derivatives.

To formalize this, suppose that $\Sigma \backslash K \approx \mathbb{R}^{3} \backslash B_{R_{0}}$, and then define

$$
\begin{aligned}
\Lambda_{\mathrm{AE}}^{0, \alpha}(\Sigma)=\left\{u: \sup |u|<\infty,\|u\|_{0, \alpha, K}<\infty,\right. \\
\left.\sup _{R \geq R_{0}} \sup _{\substack{z \neq z^{\prime} \\
R \leq|z|,\left|z^{\prime}\right| \leq 2 R}} \frac{\left|u(z)-u\left(z^{\prime}\right)\right| R^{\alpha}}{\left|z-z^{\prime}\right|^{\alpha}}<\infty\right\}
\end{aligned}
$$

$$
\Lambda_{\mathrm{AE}}^{k, \alpha}(\Sigma)=\left\{u:\|u\|_{k, \alpha, K}<\infty, \quad\left(1+|z|^{2}\right)^{\frac{|\beta|}{2}} \partial_{z}^{\beta} u \in \Lambda_{\mathrm{AE}}^{0, \alpha}, \text { for }|\beta| \leq k\right\}
$$

$$
r^{-\nu} \Lambda_{\mathrm{AE}}^{k, \alpha}(\Sigma)=\left\{u \in \Lambda_{\mathrm{AE}}^{k, \alpha}: u=r^{-\nu} v \text { on } \Sigma \backslash K \text { and } v \in \Lambda_{\mathrm{AE}}^{k, \alpha} \text { there }\right\}
$$

Thus, roughly speaking, a function $u$ is in $r^{-\nu} \Lambda_{\mathrm{AE}}^{k, \alpha}$ provided it is in the ordinary Hölder space $\Lambda^{k, \alpha}$ on any fixed compact set in $\Sigma$, and if $\left|\partial_{z}^{\beta} u\right| \leq C_{\beta}|z|^{-\nu-|\beta|}$ along the end(s), for $|\beta| \leq k$, with an appropriate condition for the Hölder derivatives of order $k+\alpha$.

We now define

$$
\mathcal{M}_{-\nu}^{k, \alpha}(\Sigma)=\left\{\text { metrics } \gamma: \gamma_{i j}-\delta_{i j} \in r^{-\nu} \Lambda_{\mathrm{AE}}^{k, \alpha}\right\}
$$

(We are abusing notation slightly here since the metric coefficients $\gamma_{i j}$ are only defined on the end $\Sigma \backslash K$.) Such metrics have been much studied in relativity, and [4 collects a number of analytic results which are appropriate for the study of the Laplacians and other natural geometric operators associated to these metrics.

In this AE setting it is natural to focus on initial data sets which would evolve into asymptotically Minkowski spacetimes, and so we assume that $\tau=0$. In addition, since

$$
\gamma \in \mathcal{M}_{-\nu}^{k+2, \alpha}(\Sigma) \Longrightarrow \operatorname{Riem}_{\gamma} \in r^{-\nu-2} \Lambda_{\mathrm{AE}}^{k, \alpha}(\Sigma),
$$

the Hamiltonian constraint equation (2) suggests that we assume that

$$
\mu \in r^{-\frac{\nu}{2}-1} \Lambda_{\mathrm{AE}}^{k, \alpha}(\Sigma)
$$

There are many other possible choices of function spaces in which to work. The most customary ones in the literature surrounding this problem are weighted Sobolev spaces. We could certainly have used these here too, but because weighted Hölder spaces have been used everywhere else in this paper it seems more natural to use spaces of this type here as well.

We now define the class of elliptic AE operators. Suppose that $P$ is an elliptic (second order) operator on $\Sigma$; we shall assume that there is a constant coefficient elliptic operator $P_{0}$ of order 2 on $\Sigma \backslash K \approx \mathbb{R}^{3} \backslash B_{R_{0}}$ such that

$$
P=P_{0}+\sum_{|\beta| \leq 2} a_{\beta}(z) \partial_{z}^{\beta}, \quad \text { where } \quad a_{\beta} \in r^{-\nu} \Lambda_{\mathrm{AE}}^{k+2-|\beta|, \alpha} .
$$


Thus by construction, if $P$ is of this type, then

$$
P: r^{-\nu} \Lambda_{\mathrm{AE}}^{k+2, \alpha}(\Sigma) \longrightarrow r^{-\nu-2} \Lambda_{\mathrm{AE}}^{k, \alpha}(\Sigma)
$$

is bounded. There are additional conditions required to ensure that this mapping is Fredholm. These are determined by the indicial roots: the number $\lambda$ is an indicial root of $P$ (on one end of $\Sigma$ ) if there exists a function $\phi(\theta), \theta=z /|z|$, such that $P\left(|z|^{\lambda} \phi(\theta)\right)=$ $\mathcal{O}\left(|z|^{\lambda-2-\nu}\right)$. Notice that no matter the value of $\lambda$ and choice of $\phi$, the right side is always of the form $P_{0}\left(r^{-\nu} \phi(\theta)\right)+\mathcal{O}\left(|z|^{\lambda-2-\nu}\right)$, where the first term here is homogeneous of degree $\lambda-2$. Hence indicial roots are determined solely by the 'principal part' $P_{0}$ of $P$. Continuing, if we express $r^{2} P_{0}$ in polar coordinates as

$$
r^{2} P_{0}=\sum_{j+\left|\beta^{\prime}\right| \leq 2} a_{j, \beta^{\prime}}\left(r \partial_{r}\right)^{j}\left(\partial_{\theta}\right)^{\beta^{\prime}}
$$

(where the coefficients $a_{j, \beta^{\prime}}$ are constant) then $\lambda$ is an indicial root for $P_{0}$ and hence for $P$ if and only if

$$
\sum_{j+\left|\beta^{\prime}\right| \leq 2} a_{j, \beta^{\prime}} \lambda^{j} \partial_{\theta}^{\beta^{\prime}} \phi(\theta)=0
$$

for some function $\phi(\theta)$. In particular, there is a denumerable set of indicial roots $\left\{\lambda_{j}\right\}$, and these are related to eigenvalues of the 'angular part' of the operator $P_{0}$.

It is known [23], 四 that if $-\nu \notin\left\{\operatorname{Re} \lambda_{j}\right\}$, then the mapping (33) is Fredholm (whereas if $-\nu$ is the real part of an indicial root, then this mapping does not have closed range). We are concerned with the question of whether this mapping is an isomorphism, or at least surjective, and if so, for which range of weights, for the particular operators $L$ and $\mathcal{L}$. It is clear from the asymptotic flatness of the data that the principal parts of $L$ and $\mathcal{L}$ are

$$
L_{0}=-\frac{1}{2} \Delta+\frac{1}{6} \nabla \operatorname{div} \quad \text { and } \quad \mathcal{L}_{0}=\Delta,
$$

respectively. (The Laplacian on the right in this expression for $L_{0}$ is the Laplacian on vector fields on $\mathbb{R}^{3}$, which is the same as the scalar Laplacian applied componentwise with respect to the standard Cartesian decomposition.) From here we compute that

- the set of indicial roots for both $L$ and $\mathcal{L}$ is $\mathbb{Z}$.

For the scalar Laplacian this is well known and derives ultimately from the fact that the global temperate solutions of $\mathcal{L}_{0} u=0$ are polynomials, and these have integer rates of growth. The negative indicial roots arise from considerations of duality between weighted $L^{2}$ spaces. Exactly the same reasoning may be applied to determine the indicial roots of $L_{0}$, for it is easy to see using the Fourier transform that the only global temperate vector fields which satisfy $L_{0} X=0$ on $\mathbb{R}^{3}$ must have polynomial coefficients.

Then we have the following

Proposition 12 When $P$ is equal to either $L$ or $\mathcal{L}$, the mapping (39) is Fredholm of index zero when $-1<-\nu<0$.

We remark that the fact that these mappings are Fredholm follows from the results quoted above and the fact that when $-\nu$ is in this range it is not an indicial root of 
either operator; the fact that they have index zero can be derived from the symmetry of these operators. When $-\nu$ is not in this range, and is not an indicial root, then the mapping (33) is still Fredholm, but no longer of index zero, hence cannot be an isomorphism.

Proposition 13 For $P=L$ or $\mathcal{L}$ and $-1<-\nu<0$, the mappings in (33) are isomorphisms.

Proof: It suffices to show that neither $L$ nor $\mathcal{L}$ have elements in their nullspace which decay like $r^{-\nu}$ as $r \rightarrow \infty$. For the linearized Lichnerowicz operator $\mathcal{L}$ this follows directly from the maximum principle. For the vector Laplacian $L$, we first note that if $X \in$ $r^{-\nu} \Lambda_{\mathrm{AE}}^{2, \alpha}(\Sigma)$ with $-1<-\nu<0$, then the boundary term in the integration by parts $\langle L X, X\rangle=\|\mathcal{D} X\|^{2}$ vanishes, and so $\mathcal{D} X=0$. We then use a result of Christodoulou [9] which says that there are no conformal Killing vector fields on AE manifolds which decay at infinity. (In fact, Christodoulou's theorem is stated only for AE manifolds which are globally diffeomorphic to $\mathbb{R}^{3}$, but one readily verifies from his proof that the result extends to general AE manifolds).

In summary, we have proved

Theorem 4 Let $(\Sigma, \gamma, \Pi)$ be an asymptotically Euclidean initial data set, as described in this section; in particular, $\tau=\operatorname{tr}_{\gamma}(\Pi)=0$ on $\Sigma$. Choose any two points $p_{1}, p_{2} \in \Sigma$, and suppose that if either of these points lies on a compact (closed) component of $\Sigma$, then there does not exist a conformal Killing field $X$ on that component which vanishes at that point, and furthermore, $\Pi \not \equiv 0$ on that component. Then there exists a one-parameter family of asymptotically Euclidean CMC initial data sets $\left(\Gamma_{T}, \Pi_{T}\right)$ on $\Sigma_{T}$ satisfying the same estimates as in Theorem 1 .

\subsection{Asymptotically hyperboloidal initial data}

Let us now turn to the case where $(\Sigma, \gamma)$ is asymptotically hyperbolic (or hyperboloidal, which is the terminology favored in the physics literature). We work within the framework of conformally compact manifolds, which is quite natural from Penrose's formulation. This is explained carefully in the introduction to [2].

Recall that a metric $\gamma$ on the interior of a manifold with boundary $\bar{\Sigma}$ is said to be conformally compact if $\gamma=\rho^{-2} \bar{\gamma}$, where $\bar{\gamma}$ is a nondegenerate metric on the closure $\bar{\Sigma}$ and $\rho$ is a defining function for $\partial \Sigma$, i.e. $\rho$ is nonnegative and vanishes precisely on the boundary, with nonvanishing differential. We shall assume that $\bar{\gamma}$ and $\rho$ are both smooth, or more accurately, polyhomogeneous (i.e. having complete expansions involving powers of a smooth defining function as well as powers of its logarithm); this latter assumption is most reasonable because it occurs generically [2]. Though we shall not do so, it is also possible to assume that $\bar{\gamma}$ and $\rho$ are in $\mathcal{C}^{k+2, \alpha}(\bar{\Sigma})$, and only minor and obvious perturbation arguments are needed to handle this more general case.

The metric $\gamma$ is complete on the interior $\Sigma$ of $\bar{\Sigma}$; moreover, assuming that $|d \rho|_{\bar{\gamma}}=1$ at $\rho=0$, then the curvature tensor $\operatorname{Riem}_{g}$ is asymptotically isotropic as $\rho \rightarrow 0$, with sectional curvatures tending to -1 . We call any such space AH (standing for either asymptotically hyperbolic or asymptotically hyperboloidal, depending on the reader's

preference). As for the tensor $\Pi=\mu+\frac{1}{3} \tau \gamma$, we assume that $\tau$ is constant, of course, and 
also that the tensor coefficients $\mu_{i j}=\mathcal{O}\left(\rho^{-1}\right)$, or equivalently $\mu^{i j}=\mathcal{O}\left(\rho^{3}\right)$. (In either case, we are writing the components of the tensor $\mu$ relative to a smooth background coordinate system on $\bar{\Sigma}$.) Assuming that $\tau=3$ and with the curvature normalization above, the constraint equations become

$$
\operatorname{div}_{\gamma}(\mu)=0, \quad R+6=|\mu|^{2} .
$$

(Recall that $|\mu|^{2}=\mathcal{O}\left(\rho^{2}\right)$.)

The existence of AH initial data sets $(\gamma, \Pi)$ satisfying these hypotheses, as well as the polyhomogeneous regularity of this data (assuming that $\bar{\gamma}$ is smooth) is the main topic of [2]. The special case of these results, in which one assumes that $\Pi$ is pure trace, is already handled in [3]. Some of the main tools required here are based on the linear analysis of geometric elliptic operators on conformally compact spaces as developed in [24], although the authors of [2] and [3] base their work on the somewhat later and less precise, although simpler methods of [1], cf. also [22] for a sharper version of these methods. The regularity theory of [2] is very closely related to the results of [25].

Quoting the main result of [2], we assume that $\Sigma$ is endowed with an $\mathrm{AH}$ initial data set $(\gamma, \Pi)$, where $\gamma$ and $\mu$ are polyhomogeneous. That is, with $\bar{\gamma}$ a smooth metric on $\bar{\Sigma}$ and $x$ a smooth defining function for $\partial \Sigma$, we have

$$
\rho \sim x+\sum_{k \geq 0} \sum_{\ell=0}^{N_{k}} a_{k \ell}(\log x)^{k} x^{\ell},
$$

and

$$
\mu_{i j} \sim \mu_{i j}^{(-1)} x^{-1}+\sum_{k \geq 0} \sum_{\ell=0}^{N_{k}} \mu_{i j}^{(k \ell)}(\log x)^{k} x^{\ell},
$$

where all coefficients $a_{k \ell}, \mu_{i j}^{(-1)}, \mu_{i j}^{(k \ell)}$ are smooth on $\bar{\Sigma}$ and for each $j$, vanish when $k>N_{j}$. (In addition, $a_{2 \ell}=0$ for $\ell>0$.)

We require the mapping properties of the vector Laplacian $L=-\operatorname{div} \circ \mathcal{D}$ and the linearized Lichnerowicz operator $\mathcal{L}=\Delta-|\mu|^{2}-3$ associated to this data. These follow directly from the general theory of [24], cf. also [22].

Choosing local coordinates $(x, y)$ near $\partial \Sigma$, then recall from these papers that any operator of the form

$$
P=\sum_{j+|\alpha| \leq 2} a_{j \beta}(x, y)\left(x \partial_{x}\right)^{j}\left(x \partial_{y}\right)^{\alpha}
$$

with coefficients $a_{j \beta}$ smooth (or just polyhomogeneous) is said to be uniformly degenerate (of order 2). Its 'uniformly degenerate symbol' is defined to be

$$
\sigma_{2}(P)(x, y ; \xi, \eta)=\sum_{j+|\beta|=2} a_{j \beta}(x, y) \xi^{j} \eta^{\beta},
$$

and $P$ is elliptic in this calculus if this symbol is nonvanishing (or an invertible matrix, if $P$ is a system) when $(\xi, \eta) \neq 0$. It is not hard to check that both of the operators $L$ and $\mathcal{L}$ above are elliptic uniformly degenerate operators.

The function spaces on which we let these operators act are the weighted Hölder spaces $x^{\nu} \Lambda_{\mathrm{AH}}^{k, \alpha}(\Sigma)$. These are taken to be the usual Hölder spaces on a compact subset of $\Sigma$ and, in a boundary coordinate chart are defined as follows: 


$$
\Lambda_{\mathrm{AH}}^{0, \alpha}(\Sigma)=\left\{u: \sup |u|<\infty, \quad \sup \frac{\left|u(x, y)-u\left(x^{\prime}, y^{\prime}\right)\right|\left(x+x^{\prime}\right)^{\alpha}}{\left(\left|x-x^{\prime}\right|+\left|y-y^{\prime}\right|\right)^{\alpha}}<\infty\right\}
$$

$$
\Lambda_{\mathrm{AH}}^{k, \alpha}(\Sigma)=\left\{u:\left(x \partial_{x}\right)^{j}\left(x \partial_{y}\right)^{\beta} u \in \Lambda_{\mathrm{AH}}^{0, \alpha}, \quad j+|\beta| \leq k\right\}
$$

$$
x^{\nu} \Lambda_{\mathrm{AH}}^{k, \alpha}(\Sigma)=\left\{u=x^{\nu} v: v \in \Lambda_{\mathrm{AH}}^{k, \alpha}\right\} .
$$

Clearly

$$
P: x^{\nu} \Lambda_{\mathrm{AH}}^{k+2, \alpha}(\Sigma) \longrightarrow x^{\nu} \Lambda_{\mathrm{AH}}^{k, \alpha}(\Sigma)
$$

is bounded for any $\nu$, but just as in the AE case, restrictions on $\nu$ must be imposed to ensure that this map is Fredholm.

To state these, we say that a number $\lambda$ is an indicial root for $P$ if $P x^{\lambda}=\mathcal{O}\left(x^{\lambda+1}\right)$ as $x \rightarrow 0$. It is always true that $P x^{\lambda}=\mathcal{O}\left(x^{\lambda}\right)$ and the indicial root condition requires that $\lambda$ satisfy a polynomial equation involving the coefficients $a_{j 0}(0, y)$. (It is possible that these roots could depend on $y$, but in our application this does not occur.) A brief calculation, using that $|\mu|^{2} \rightarrow 0$ as $x \rightarrow 0$, gives:

- The indicial roots for $L$ are 0 and 4 ,

- The indicial roots for $\mathcal{L}$ are -1 and 3 .

We now state one of the main theorems from [24], specialized to this particular setting:

Proposition 14 Suppose that $0<\nu<4$ and $-1<\nu^{\prime}<3$. Then the mappings

$$
L: x^{\nu} \Lambda_{A H}^{k+2, \alpha}(\Sigma) \longrightarrow x^{\nu} \Lambda_{A H}^{k, \alpha}(\Sigma)
$$

and

$$
\mathcal{L}: x^{\nu^{\prime}} \Lambda_{A H}^{k+2, \alpha}(\Sigma) \longrightarrow x^{\nu^{\prime}} \Lambda_{A H}^{k, \alpha}(\Sigma)
$$

are Fredholm of index 0. Furthermore, the nullspace of either of these operators is independent of $\nu \in(0,4)$ and $\nu^{\prime} \in(-1,3)$.

To conclude that these maps are isomorphisms, it suffices to prove

Proposition 15 For $\nu$ and $\nu^{\prime}$ in the ranges stated in the previous proposition, the maps $L$ and $\mathcal{L}$ are injective.

Proof: For $\mathcal{L}$ this is quite easy. In fact, if $\mathcal{L} \phi=0$, then $|\phi| \leq C x^{\nu^{\prime}}$ for any $\nu^{\prime} \in(-1,3)$, and therefore $\phi \rightarrow 0$ at $\partial \Sigma$. But the terms of order 0 in $\mathcal{L}$ are strictly negative, and so the maximum principle implies that $\phi \equiv 0$. As for $L$, we note that if $L X=0$, then the coefficients of $X$ are $\mathcal{O}\left(x^{4}\right)$, and so we may perform the usual integration by parts to obtain that $\mathcal{D} X=0$. By the conformal invariance of the nullspace of $\mathcal{D}=\mathcal{D}_{\gamma}$, we also have that $\mathcal{D}_{\bar{\gamma}} X=0$. Thus $X$ is a (smooth or polyhomogeneous) conformal Killing vector field for the metric $\bar{\gamma}$ on $\bar{\Sigma}$, with coefficients vanishing to order 4 at the boundary. Since we already know that $X$ is polyhomogeneous, the equation $\mathcal{D}_{\bar{\gamma}} X=0$ now implies that $X$ vanishes to infinite order as $x \rightarrow 0$, and it is known that no such conformal Killing field can exist [8]. Thus we have shown that 
Proposition 16 The mappings in Proposition 14 are both isomorphisms.

Therefore, we may use the spaces occuring here as the spaces $E, F, E^{\prime}$ and $F^{\prime}$ needed in Propositions 10 and 11. This proves

Theorem 5 Suppose that $(\Sigma, \gamma, \Pi)$ is an asymptotically hyperbolic initial data set, so that $\gamma$ is a conformally compact metric and $\tau=\operatorname{tr}_{\gamma}(\Pi) \equiv 3$. Let $p_{1}, p_{2} \in \Sigma$, and suppose that if either of these points lies on a compact (closed) component of $\Sigma$, then there does not exist a conformal Killing field $X$ on that component which vanishes at that point. Then there exists a one-parameter family of asymptotically hyperbolic CMC initial data sets $\left(\Gamma_{T}, \Pi_{T}\right)$ on the manifold $\Sigma_{T}$ satisfying the same estimates as in Theorem 1 .

\section{The geometry of the neck}

We have now shown in the three main settings ( $\Sigma$ compact or $\mathrm{AE}$ or $\mathrm{AH}$ ) that it is possible to produce one-parameter families of CMC initial data sets $\left(\Gamma_{T}, \Pi_{T}\right)$ which are arbitrarily good approximations to the original initial data set $(\gamma, \Pi)$ away from the points $p_{1}, p_{2}$. As $T \rightarrow \infty$, various geometric quantities associated to these tensors degenerate in the neck region. We describe this in more detail now.

We shall only consider quantities which depend on at most two derivatives of the metric, and so we fix any $k \geq 2$ and $\alpha \in(0,1)$ and construct the initial data sets as in $\S \S 2-7$.

Recall the notation $\Sigma_{R}^{*}=\Sigma \backslash\left(B_{R}\left(p_{1}\right) \cup B_{R}\left(p_{2}\right)\right)$. Next, for $b \in(0, R)$, let $A_{j}(b)=$ $B_{R}\left(p_{j}\right) \backslash B_{b}\left(p_{j}\right)$, and finally denote by $C_{T, b}$ the part of the neck region not covered by these annuli. Then for each $T>0$,

$$
\Sigma_{T}=\Sigma_{R}^{*} \sqcup\left(A_{1}(b) \cup A_{2}(b)\right) \sqcup C_{T, b} .
$$

In either of the annuli and in the truncated neck region we shall use the $(s, \theta)$ coordinates, so that, for example, $A_{1}(b)=\{T / 2-\beta \leq s \leq T / 2\} \times S^{2}$ and $C_{T, b}=\{-T / 2+\beta \leq s \leq$ $T / 2-\beta\} \times S^{2}$, for $\beta=-\log b$. Write $\psi_{T}^{4} \gamma_{T}=\tilde{\gamma}_{T}$; of course, this metric is the same as $\gamma$ in $\Sigma_{R}^{*}$, and the deviation between these two metrics in either of the half-necks $0<|s|<T / 2$ is $\mathcal{O}\left(\left(e^{-T} \cosh s\right)^{2}\right)$. We shall estimate $\left|\Gamma_{T}-\tilde{\gamma}_{T}\right|_{\tilde{\gamma}_{T}}$ (i.e. the difference between the actual solution $\Gamma_{T}$ and the approximate solution $\left.\tilde{\gamma}_{T}\right)$ in each of these regions.

By definition, $\Gamma_{T}=\left(\psi_{T}+\eta_{T}\right)^{4} \gamma_{T}$. Hence

$$
\left|\Gamma_{T}-\tilde{\gamma}_{T}\right|_{\tilde{\gamma}_{T}} \approx \psi_{T}^{-4}\left(\left(\psi_{T}+\eta_{T}\right)^{4}-\psi_{T}^{4}\right) \leq C\left|\eta_{T} / \psi_{T}\right| .
$$

Recall also from $\S$ 囵 that $\left|\eta_{T}\right| \leq \nu e^{-T / 4} w_{T}^{\delta}$, Furthermore, $1 / 2 \leq w_{T} / \psi_{T} \leq 2$ everywhere. Therefore:

- In $\Sigma_{R}^{*}, \tilde{\gamma}_{T}=\gamma$ and $\psi_{T} \approx 1$. Thus

$$
\left|\Gamma_{T}-\tilde{\gamma}_{T}\right|_{\tilde{\gamma}_{T}} \leq C e^{-T / 4}
$$

in this region.

- In all of $C_{T}$, we have

$$
\left|\Gamma_{T}-\tilde{\gamma}_{T}\right|_{\tilde{\gamma}_{T}} \leq C e^{-T / 4}\left(e^{-T / 4} \cosh (s / 2)\right)^{\delta-1}=C e^{-\delta T / 4}(\cosh (s / 2))^{\delta-1} .
$$


- In particular, in $A_{1}(b),\left|\eta_{T}\right| \leq \nu e^{-T / 4} w_{T}^{\delta}$, and so

$$
\left|\Gamma_{T}-\tilde{\gamma}_{T}\right|_{\tilde{\gamma}_{T}} \leq C e^{-T / 4}\left(e^{-T / 4} e^{s / 2}\right)^{\delta-1}=C e^{-\delta T / 4} e^{(\delta-1) s / 2} \leq C e^{(1-\delta) \beta / 2-T / 4} .
$$

The estimate in $A_{2}(b)$ is identical.

Notice in each of these cases that since $\delta<1$, this difference of tensors is much smaller than $\tilde{\gamma}_{T}$, and therefore geometric quantities relative to $\Gamma_{T}$ are very well approximated by the same quantities relative to $\tilde{\gamma}_{T}$.

The following two results are immediate corollaries.

Proposition 17 Let $c$ be any $\mathcal{C}^{1}$ path in $\Sigma_{T}$ which runs through the neck region. For simplicity we shall assume that it has the simple form $s \mapsto\left(s, \theta_{0}\right),-T / 2 \leq s \leq T / 2$. Then

$$
\operatorname{length}_{\Gamma_{T}}(c) \sim \operatorname{length}_{\tilde{\gamma}_{T}}(c)+\mathcal{O}\left(e^{-\delta T / 4}\right) .
$$

In particular, for the portion of $c$ which lies in either of the annuli $A_{j}(b)$, we may substitute length ${ }_{\gamma}(c)$ for length $\tilde{\gamma}_{T}(c)$ on the right side here.

Proposition 18 In $C_{T, b}$, the full curvature tensor $\operatorname{Riem}_{\Gamma_{T}}$ satisfies

$$
\left|\operatorname{Riem}_{\Gamma_{T}}\right|_{\tilde{\gamma}_{T}} \leq C \psi_{T}^{-4} \leq C e^{T}(\cosh s)^{-2} .
$$

In particular, the scalar curvature $R_{\Gamma_{T}}$ satisfies this same estimate.

Of course, we could also compute finer asymptotics for the full curvature tensor and for the scalar curvature function.

Estimates for the extrinsic curvature tensor are also easy to obtain. We have already shown that the deviation of the trace-free part of $\Pi_{T}$ from that of $\Pi$ on $\Sigma_{R}^{*}$ is bounded by $T^{3} e^{-3 T / 2}$. On the other hand, relying on the fact that $|\mu|_{\gamma_{c}} \leq C e^{-T / 2} \cosh s$ together with the preceding estimates, we see that

$$
\left|\Pi_{T}\right|_{\Gamma_{T}} \leq C e^{T / 2}(\cosh s)^{-1}, \quad \text { on } \quad C_{T} .
$$

We may use this as a check on this last proposition: the constraint equation (2) gives the scalar curvature in terms of $\left|\Pi_{T}\right|_{\Gamma_{T}}^{2}$, and we see that the estimates are the same. However, we also see from this that if the original tensor $\mu=0$ in both balls $B_{1}$ and $B_{2}$, then $\left|\Pi_{T}\right|_{\Gamma_{T}}$ is bounded in $C_{T}$, and hence $R_{\Gamma_{T}}$ is also.

\section{$9 \quad$ Examples and applications}

There are a number of constructions and applications of special interest in general relativity to which our results pertain. In this section we briefly describe several of these. This discussion is intended to be informal and descriptive, and so we do not present these results formally, but given the rest of the contents of this paper, it should be obvious how to do so. These examples are separated into different subsections, but these divisions are artificial and are intended for guidance only. 


\section{Adding wormholes}

The first construction is that of adding a physical wormhole to (almost) any globally hyperbolic solution of the vacuum (Lorentzian) Einstein equations which admits a CMC Cauchy surface. One proceeds by: a) choosing such a Cauchy surface $\Sigma$; b) choosing any pair of points on $\Sigma$; c) verifying the conditions of Theorems 1 , 4 or 5 ; d) carrying out the gluing construction as outlined in $\$ \S 2-6$; and finally, e) evolving the resulting initial data. Standard well-posedness theorems [11], [13] guarantee that there is a unique (up to spacetime diffeomorphism) maximal spacetime development of this initial data set. In other words, there is a spacetime which satisfies the vacuum Einstein equations, has the topology $\Sigma_{T} \times \mathbb{R}$, restricts to the wormhole initial data on $\Sigma_{T} \times\{0\}$, and also contains every other spacetime which satisfies these three properties. Of course, this spacetime will probably not last very long, and indeed it is likely that the evolving wormhole will pinch off quickly, thus preventing the spacetime from evolving further in time. Nonetheless, there is a spacetime solution which evolves from this initial data and which therefore contains a spacetime wormhole connecting the chosen points; in addition, the evolving spacetime remains essentially unchanged from its previous unglued state outside the domain of influence of the initial data on the wormhole region.

There do exist spacetimes for which this wormhole addition cannot be carried out: these are the ones which do not carry any set of CMC data which matches the hypotheses of our theorems. A simple example of this phenomenon is the 'spatially compact Minkowski space' $T^{3} \times \mathbb{R}$, since all CMC slices of this spacetime are compact and have vanishing extrinsic curvature, and hence the hypotheses of Theorem 1 are not satisfied.

\section{Multiple black hole spacetimes}

Over 40 years ago Misner [30] constructed a number of explicit initial data sets involving two asymptotically Euclidean regions connected by a wormhole. Soon thereafter, he and others, cf. [17], recognized that since the wormhole inevitably contains a minimal two-sphere, and since the presence of such a two-sphere necessitates the existence of an apparent horizon, the spacetime developments of these initial data sets must contain black holes. In subsequent work, Misner [31 constructed explicit (series form) sets of initial data with more than one wormhole; the spacetime developments of these data must then contain more than one black hole. Misner's data has been used extensively by numerical relativists [10] in studying multi-black hole spacetimes.

Our gluing constructions yield a much wider class of multi-black hole solutions. Most importantly, we can successively add black holes to any given asymptotically flat spacetime. (We say that a spacetime is asymptotically flat if it is foliated by asymptotically Euclidean initial data sets; see chapter 11 in [34] for a more rigorous definition.) The idea is as follows: start with any asymptotically flat spacetime which admits a maximal (i.e. zero mean curvature), asymptotically Euclidean hypersurface. Choose such a maximal Cauchy surface with $\mathrm{AE}$ data $(\Sigma, \gamma, \Pi)$ and a point on this hypersurface, and then glue on a copy of Euclidean space $\mathbb{R}^{3}$ with zero extrinsic curvature (which is a maximal data set for Minkowski space). The result is a new initial data set with two AE regions connected by a wormhole, with the data largely unchanged on either side of the wormhole. As before, the wormhole contains a minimal two-sphere, and so black hole formation is inevitable in the spacetime development of this data. We may now continue this process, taking this new data and gluing on a further copy of maximal data for Minkowski space. 
This yields a spacetime with two black holes. Proceeding further, we obtain spacetimes with an arbitrary (finite, or even infinite) number of black holes, with each additional black hole leaving the data for the previous black holes largely unchanged.

If one glues two AE sets of data together, the resulting data set has two AE ends. From a mathematical point of view, there is nothing to distinguish one of these ends from the other. However, in using Einstein solutions to model spacetime physics, one often makes a choice of one of the ends, and its spacetime development, to be the physically accessible "exterior", with the other end corresponding to a physically inaccessible "interior". The exterior is the home of the "observers at infinity" while the interior is somewhere "inside the black hole". Apart from considerations of physical modelling, the choice is arbitrary.

Multi-black hole spacetimes have several ends, and any one of these may be regarded as the exterior. However, to model spacetimes with observable interacting black holes, one must construct multi-black hole initial data sets with the chosen exterior asymptotic region directly connected to each of the wormholes. Each of these wormholes leads through to its opposite interior end. These interiors may be independent of one another, or they may coincide as a single asymptotic region. In other words, one may envision the manifold as having, for example, two asymptotic regions connected by several necks. This case corresponds to Misner's "matched throat" data sets [31], and is no more difficult to construct from our point of view than a solution with independent interior ends.

We emphasize that the multi-black hole data sets we are describing here are not presented as explicitly as Misner's examples, because to obtain them one must solve the constraint equations in conformal form. In practice, there are efficient ways to do this numerically. As already noted, one virtue of our results is that we obtain solutions which leave the spacetime essentially unchanged away from the domain of influence of the added black hole. This feature is quite interesting and should be useful to numerical relativists.

\section{Adding AE ends to closed initial data sets}

Every closed three manifold $\Sigma$ admits a CMC solution of the vacuum constraint equations: we choose $\gamma$ to be a metric of constant negative scalar curvature $R(\gamma)=-6$ (these always exist), and set $\Pi=\gamma ;(\Sigma, \gamma, \gamma)$ is then a solution of the constraint equations, with constant mean curvature $\tau=3$. It is of interest to know whether every manifold of the form $\Sigma \backslash\{p\}$ with $\Sigma$ closed admits AE initial data sets. For a manifold $\Sigma$ which admits a metric of positive scalar curvature with no conformal Killing fields and with a nonvanishing symmetric transverse-traceless 2-tensor, our methods show that this is the case. To see this, we first use [18] to get that any such $\Sigma$ admits a solution $(\Sigma, \gamma, \Pi)$ of the constraint equations with $\tau=0$. Then by Theorem 1 , we may glue the standard AE initial data set on $\mathbb{R}^{3}$ with vanishing extrinsic curvature to $(\Sigma, \gamma, \Pi)$ to get an AE initial data set on $\Sigma \backslash\{p\}$. Note the closely related result, that when $\Sigma$ is compact, $\Sigma \backslash\{p\}$ admits a complete scalar flat metric, i.e. a time-symmetric AE initial data set, if and only if $\Sigma$ admits a metric of positive scalar curvature, cf. [25].

On the other hand, suppose that $\Sigma$ has the property that any metric of nonnegative scalar curvature on it is flat (for example, $\Sigma=T^{3}$ has this property). Then by (2), any initial data set $(\Sigma, \gamma, \Pi)$ with $\tau=0$ has nonnegative scalar curvature. By the hypothesis on $\Sigma$, this means that $\gamma$ is flat, and so, using (2) again, we must have $\Pi \equiv 0$. But $(\Sigma, \gamma, 0)$ does not satisfy the hypotheses of Theorem 4 , and so we cannot use our gluing theorem to produce AE initial data sets on $\Sigma \backslash\{p\}$. 
We now comment briefly on the physical interpretation of the solutions we obtain by gluing $\mathrm{AE}$ ends to closed initial data sets as above. Suppose we view the compact side as the exterior, accessible region. This data has a minimal two-sphere as before, and therefore most likely evolves into a spacetime with an apparent horizon. But the problem is that there is no clear and unambiguous definition of a black hole relative to an exterior spacetime region which is not asymptotically flat. This makes the interpretation of the spacetime development in this case less transparent, but it also makes such spacetimes rather interesting. Indeed, one might be able to learn a lot about how to work with black holes in spatially closed spacetimes by careful study of these examples.

As noted above, if a spacetime contains a wormhole connecting a pair of regions, one may choose either of the pair of regions as the exterior accessible region. The choice is mathematically arbitrary, but physically important for modelling. Thus the spacetimes one obtains by gluing together data from an asymptotically flat spacetime and data from a spatially compact spacetime, and then evolving, may be viewed either as a spatially compact cosmology with a black hole-like object in it (with the closed region as the exterior), or as a black hole with a nontrivial interior solution. The construction is the same; all we are doing is redefining which region we consider to be the exterior, physical region. The wormhole may cut off very quickly and render the interior solution physically irrelevant; this is the nature of black hole physics, and we believe that it can be studied meaningfully with these gluing constructions.

\section{Adding AH ends to closed initial data sets}

In distinction to the $\mathrm{AE}$ case, since $\mathrm{AH}$ initial data sets have nonvanishing mean curvature $\tau=3$, it is possible to glue an $\mathrm{AH}$ initial data set to an appropriately chosen intial data set on any compact manifold $\Sigma$. In fact, as above, let $\gamma$ be a metric of constant negative scalar curvature $R=-6$ on $\Sigma$, so that $(\Sigma, \gamma, \gamma)$ is an initial data set also with $\tau=3$. By Theorem 5, we may glue to it any AH initial data set (in particular, the hyperboloid $\left(\mathbb{H}^{3}, \gamma_{0}, \gamma_{0}\right)$, where $\gamma_{0}$ is the standard hyperbolic metric) at any point $p \in \Sigma$. This proves in particular that for every closed manifold $\Sigma$, the punctured manifold $\Sigma \backslash\{p\}$ admits an $\mathrm{AH}$ initial data set. In fact, we may iterate this procedure and obtain $\mathrm{AH}$ initial data sets on $\Sigma \backslash\left\{p_{1}, \ldots, p_{k}\right\}$ for any collection of distinct points on any closed 3 -manifold $\Sigma$. We note again that the analogue of this question for AE vacuum initial data sets is still open.

As a simple and interesting example of this last construction, let $(\Sigma, \gamma)$ be a closed hyperbolic 3-manifold. By adjoining to it the hyperboloid as above we obtain an $\mathrm{AH}$ initial data set on $\Sigma \backslash\{p\}$. In fact, we may find such initial data sets which are arbitrarily small perturbations of the initial hyperbolic metric away from the neck.

It is also straightforward to glue together two or more AH data sets. Not surprisingly, it is impossible to glue CMC AH data sets to CMC AE data sets because the constant mean curvatures in the two cases are different.

We are left with the question of whether the spacetimes which result from evolving these glued AH initial data sets develop black holes. Again, if we view the spatially compact part of the data as the exterior, physically accessible, part of the solution, then this question does not have a definite meaning, since as before, black holes are only well-defined in spacetimes with asymptotically flat ends. 


\section{References}

[1] L. Andersson, Elliptic systems on manifolds with asymptotically negative curvature, Indiana Univ. Math. Jour. 42 (1993) No. 4, 1359-1387.

[2] L. Andersson and P. T. Chruściel, Solutions of the constraint equations in general relativity satisfying "hyperboloidal boundary conditions", Dissertationes Math. (Rozprawy Mat.) 355 (1996)

[3] L. Andersson, P. T. Chruściel, H. Friedrich, On the regularity of solutions to the Yamabe equation and the existence of smooth hyperboloidal initial data for Einstein's field equations, Comm. Math. Phys. 149 (1992), no. 3, 587-612.

[4] R. Bartnik, The mass of an asymptotically flat manifold, Comm. Pure Appl. Math. 39 (1986) 661-693.

[5] R. Bartnik, Remarks on cosmological spacetimes and constant mean curvature surfaces, Comm. Math. Phys. 117 (1988) no. 4, 615-624.

[6] D. Brill, On spacetimes without maximal slices in "Proceedings of the Third Marcel Grossman Meeting", H Ning, ed. Science Press (1983) 79-87

[7] M. Cantor, The existence of non-trivial asymptotically flat initial data for vacuum spacetimes, Comm. Math. Phys. 57 (1977), no. 1, 83-96.

[8] D. Christodoulou and N. O'Murchadha, The boost problem in general relativity Comm. Math. Phys. 80 (1981) 271-300.

[9] D. Christodoulou and Y. Choquet-Bruhat, Elliptic systems in $H_{s, \delta}$ spaces on manifolds which are Euclidean at infinity, Acta. Math. 146 (1981) 129-150.

[10] G. Cook, Initial data for numerical relativity, Living Reviews (2000), 2000-2005.

[11] Y. Choquet-Bruhat, Théorème d'existence pour certains systèmes d'équations aux dérivées partialles non linéaires, Acta. Math. 88 (1952) 141-225.

[12] Y. Choquet-Bruhat, Solution of the coupled Einstein constraints on asymptotically Euclidean manifolds in "Directions in General Relativity", Vol. 2, B. L. Hu and T. A. Jacobson ed. Cambridge University Press, Cambridge (1993) 83-96.

[13] Y. Choquet-Bruhat and R. Geroch, Global aspects of the Cauchy problem in general relativity, Comm. Math. Phys. 14 (1969), 329-335.

[14] Y. Choquet-Bruhat, J. Isenberg and J. York, Einstein constraints on asymptotically Euclidean manifolds, Phys. Rev. D (3) 61 (2000), no. 8, 084034, 20 pp.

[15] Y. Choquet-Bruhat and J. York, The Cauchy Problem, in General Relativity, A. Held ed., Plenum 1979.

[16] J. Corvino, Scalar curvature deformation and a gluing construction for the Einstein constraint equations, Comm. Math. Phys. 214 (2000), no. 1, 137-189. 
[17] S. Hawking and G. Ellis, The Large Scale Structure of Space-time, Cambridge Univ Press, (1973).

[18] J. Isenberg, Constant mean curvature solutions of the Einstein constraint equations on closed manifolds, Class. Quantum Grav. 12 (1995) 2249-2274.

[19] J. Isenberg and V. Moncrief, A set of nonconstant mean curvature solutions of the Einstein constraint equations on closed manifolds,. Class. Quantum Grav. 13 (1996) 1819-1847.

[20] J. Isenberg and J. Park, Asymptotically hyperbolic non-constant mean curvature solutions of the Einstein constraint equations, Class. Quantum Grav. 14 (1997) A189-A201.

[21] N. Kapouleas, Complete constant mean curvature surfaces in Euclidean three space, Ann. of Math. (2) 131 (1990), 239-330.

[22] J. Lee, Fredholm operators and Einstein metrics on conformally compact manifolds, arXiv:math.DG/0105046.

[23] R. Lockhart and R. McOwen, On elliptic systems in $\mathbb{R}^{n}$, Acta Math. 150 (1983), no. 1-2, 125-135.

[24] R. Mazzeo, Elliptic theory of differential edge operators, I, Comm. Partial Diff. Eqns. 16 (1991), no. 10, 1615-1664.

[25] R. Mazzeo, Regularity for the singular Yamabe problem, Indiana Univ. Math. Jour. 40 (1991), no. 4, 1277-1299.

[26] R. Mazzeo and F. Pacard Constant scalar curvature metrics with isolated singularities, Duke Math. Journal 99 No. 3 (1999) 353-418.

[27] R. Mazzeo and F. Pacard Constant mean curvature surfaces with Delaunay ends, Comm. Anal. Geom. 9 No. 1 (2001) 169-237.

[28] R. Mazzeo, F. Pacard and D. Pollack Connected Sums of constant mean curvature surfaces in Euclidean 3 space, J. Reine Angew. Math. 536 (2001), 115-165.

[29] R. Mazzeo, D. Pollack and K. Uhlenbeck, Connected sum constructions for constant scalar curvature metrics, Topol. Methods Nonlinear Anal. 6 (1995), no. 2, 207-233.

[30] C.W. Misner, Wormhole initial conditions, Phys. Rev. 118 (1960), 1110-1111.

[31] C.W. Misner, The method of images in geometrostatics, Ann. Phys. 24 (1963), 102-117.

[32] J. Rosenberg and S. Stolz, Manifolds of positive scalar curvature, in "Algebraic Topology and its Applications" 27 (1994) G.E. Carlsson et al., eds., Math. Sci. Res. Inst. Publ. Springer-Verlag, NY, 241-267.

[33] M. Taylor, Partial differential equations III: nonlinear equations, Appl. Math. Sci. 117 (1996), Springer-Verlag, New York. 
[34] R. Wald, General Relativity, Univ. Chicago Press, Chicago (1984). 